TRABAJOS DE PREHISTORIA

66, N. ${ }^{\circ}$ 1, enero-junio 2009, pp. 47-74, ISSN: 0082-5638

doi: $10.3989 /$ tp.2009.09012

\title{
Comportamiento económico de los últimos cazadores-recolectores y primeras evidencias de domesticación en el occidente de Asturias. La Cueva de Mazaculos II
}

\author{
Economic behaviour of the last hunter-gatherers and the first evidence for domestication \\ in Western Asturias. The Cave of Mazaculos II
}

\author{
Ana Belén Marín Arroyo (*) \\ Manuel Ramón González Morales (**)
}

\section{RESUMEN}

El estudio del comportamiento económico desarrollado por los últimos grupos de cazadores-recolectores del Mesolítico Cantábrico, fundamentalmente dominado por el abundante registro de la explotación del medio marino, cuenta con reducidas evidencias del consumo de mamíferos terrestres, en lo que supone una ruptura con la tendencia observada durante el Paleolítico Superior. Las causas de este cambio y su implicación en la demografía de la región se investigan en este trabajo mediante el análisis arqueozoológico y tafonómico detallado de la macrofauna del yacimiento de Mazaculos II (Ribadedeva, Asturias), un conchero que alberga uno de los depósitos fósiles más importantes del período. Adicionalmente se presentan los primeros indicios de domesticación en el Cantábrico occidental.

\begin{abstract}
The study of the economic behaviour of the last hunter-gatherers of the Cantabrian Mesolithic, mainly dominated by the rich assemblages related to marine exploitation, has limited evidences of terrestrial mammals consumption, which implies a break with the general trend observed during the Upper Palaeolithic. The reasons behind this change and its implication in the demog-
\end{abstract}

(*) Leverhulme Centre for Human Evolutionary Studies. University of Cambridge. The Henry Wellcome Building. Fitzwilliam Street. CB2 1QH Cambridge - United Kingdom. Correo electrónico: abm38@cam.ac.uk

(**) Instituto Internacional de Investigaciones Prehistóricas de Cantabria. Universidad de Cantabria. Av. Los Castros, s/n. 39005 Santander. Correo electrónico: moralesm@unican.es

Recibido: 31-X-2008; aceptado: 6-III-2009. raphy of the region are assessed here with the detailed archaezoological and taphonomical analysis of the macromammals of Mazaculos II Cave (Ribadedeva, Asturias), a shell-midden that houses one of the most important fossil deposits of this period. In addition the first signs of domestication in Western Cantabria are presented.

Palabras clave: Cantábrico; Mazaculos II; Mesolítico; domesticación; Arqueozoología; Demografía.

Key words: Cantabrian Spain; Mazaculos II; Mesolithic; Domestication; Archaeozoology; Demography.

\section{INTRODUCCIÓN}

El registro arqueológico de la Cornisa Cantábrica, con uno de los conjuntos más ricos del continente europeo durante gran parte del Paleolítico Superior (Straus 2005), evidencia, tras la llegada del Holoceno, un cambio significativo en la organización económica, social y territorial de los grupos cazadores-recolectores que la habitaron, manifestada en una aparente simplificación tecnológica (Clark 1995; Fernández-Tresguerres 1983; González Morales 1995a; Straus 1992), una polarización del hábitat hacia la zona litoral (Fano 1996; González Morales 1999; Straus y González Morales 2003) y un supuesto empobrecimiento productivo (Clark 2004; Straus 1995, 2004).

Así, a pesar de la intensa labor de investigación desarrollada durante las tres últimas décadas (Arias 1991; Arias y Pérez 1995; Arias et al. 
2007a, 2007b; Berganza 1990; Clark 1976; Fano 1998; González Morales 1982, 2000; González Morales y Díaz Casado 1991/91; Muñoz y Berganza 1997; Ontañón et al. 2000; Pérez Suárez 1995; Ruiz Cobo y Smith 2001), la mayor parte de los yacimientos descubiertos hasta la fecha corresponden a la tipología de "concheros", en pequeñas y medianas cavidades costeras, dominados por un extenso registro de malacofauna y sencillas herramientas líticas de cuarcita (Vega del Sella 1923). Además de las implicaciones que esto tiene en la evolución cultural de las sociedades mesolíticas desde un punto de vista exclusivamente económico, destaca significativamente la escasez de restos de mamíferos terrestres (en especial de ungulados) en contraste con momentos del Paleolítico Superior (Fano 2004; Straus 2004).

Bien como consecuencia de un uso marginal de estos yacimientos por parte de grupos humanos asentados en campamentos al aire libre no conservados (Straus 1979, 1992), como producto de decisiones económicas humanas en un contexto de presión demográfica creciente (Clark y Straus 1983, 1986; Clark y Lerner 1980; Clark 1987, 2004; Straus et al. 1980), como fruto de una crisis alimenticia severa derivada de una reducción súbita de los recursos disponibles (Cueto et al. 2005/ 2006; Estévez 2005) o como resultado de una visibilidad diferencial de yacimientos debida a la transgresión marina (Bailey 1978, 1983; Fano, 1996, 2004; González Morales 1982) no se dispone de un registro faunístico adecuado para analizar aspectos relevantes de los últimos grupos cazadores-recolectores cantábricos, así como la estrategia de subsistencia desarrollada, la funcionalidad y estacionalidad de sus asentamientos o los primeros indicios de cambio hacia sociedades agropastoriles. A esto hay que añadir el problema que representan algunas excavaciones antiguas (p.e. Cueto de la Mina, Penicial, Coberizas), que únicamente ofrecen datos cualitativos, o la frecuente ausencia de análisis tafonómicos completos.

Asimismo, en lo que se refiere al proceso de neolitización subsiguiente, la escasez de evidencias arqueozoológicas durante los inicios de este fenómeno ha dificultado históricamente el conocimiento de esta transición, a menudo caracterizada por el hallazgo de restos cerámicos (Arias y Pérez 1995), con las precauciones que esto implica (Fano 2000). Sin embargo, las evidencias económicas más sólidas de la adopción de los prime- ros rasgos agrícolas o ganaderos se localizan en El Mirón (5790 \pm 90 BP) (Peña-Chocarro et al. 2005), en Los Gitanos (5771 \pm 499 BP) (Ontañón 2005), en Arenaza (5755 \pm 65 BP) (Altuna 1979, Arias y Altuna 1999), Kobaederra (5820 \pm 240 BP) (Zapata et al. 1997), Herriko Barra (Iriarte et al. 2005) y en Marizulo (5285 \pm 65 BP) (Altuna 1980) si bien, el registro sigue siendo exiguo para poder responder a la incertidumbre existente sobre el origen de estas conductas, existiendo actualmente hipótesis exógenas (González Morales 1992) o endógenas (Arias 1991).

En este contexto, el presente trabajo recoge los resultados del análisis arqueozoológico efectuado a los restos de macromamíferos de los niveles mesolíticos y neolíticos del yacimiento de Mazaculos II, situado en las proximidades de La Franca, en Ribadedeva (Asturias). Este yacimiento, excavado inicialmente por Vega del Sella en 1915 y reexcavado con una metodología moderna en los años setenta y ochenta (González Morales 1978, 1995b; González Morales y Márquez Uría 1978; Gónzalez Morales et al. 1980), supuso ya un cambio en la interpretación del período de estudio, aportando pruebas de un uso residencial de un conchero y refutando las hipótesis de una complementariedad costa-interior entre las culturas arqueológicas asturiense y aziliense (González Morales 1982).

En esta ocasión, el empleo de técnicas tafonómicas y estadísticas avanzadas, ha permitido obtener nueva información sobre la organización económica de los grupos mesolíticos y la funcionalidad de los concheros por ellos generados, apoyando la teoría de la existencia de una ruptura con los antecedentes azilienses en algún momento de la transición Pleistoceno-Holoceno. Las consecuencias de este desequilibrio, manifestadas en un posible descenso demográfico acusado, podrían apoyar también la hipótesis de una posterior llegada externa de los comportamientos productores. Asimismo, se han reconocido las primeras evidencias osteológicas de domesticación animal en la zona asturiana durante el $\mathrm{V}$ milenio.

\section{MATERIALES Y MÉTODOS}

\subsection{La Cueva de Mazaculos II}

El yacimiento de Mazaculos recibe su nombre de una finca situada sobre un pequeño macizo 


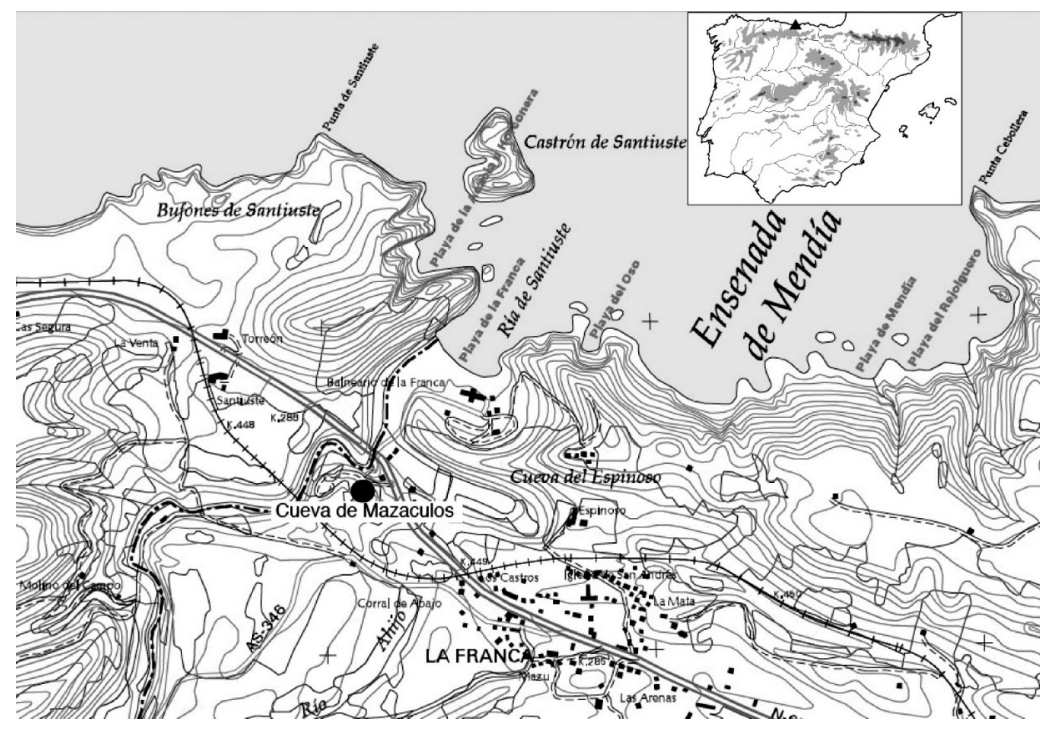

Fig. 1. Ubicación del yacimiento de Mazaculos II.

calcáreo que domina la desembocadura del río Cabra en la ría de La Franca (Ribadedeva, Asturias) (Fig. 1). Sus coordenadas UTM son $\mathrm{x}=372.135 ; \mathrm{y}=4.805 .485$ y la boca se sitúa a una altitud de $35 \mathrm{~m}$ sobre el nivel medio de las pleamares locales.

El abrigo exterior, situado en el fondo de un anfiteatro calizo de unos $10 \mathrm{~m}$ de altura, está orientado al NNO (Lám. I), si bien se encuentra protegido parcialmente de los vientos de ese cuadrante por algunos relieves cercanos. La anchura varía entre 16 y $5 \mathrm{~m}$ y el suelo buza unos $25^{\circ}$ hacia el SE.
En el extremo ESE, el abrigo conecta con una sala interior de amplias proporciones y techo elevado, que se adentra unos $25 \mathrm{~m}$ en el macizo y que cuenta con los únicos testimonios de arte parietal. Un nuevo pasaje de techo muy bajo da paso a una segunda sala más reducida, situada al NE de la primera (Lám. I).

Las labores de excavación, desarrolladas entre los años 1976 y 1983, abarcaron tanto el abrigo exterior, sobre un amplio conchero asturiense, como la zona interior, con niveles arqueológicos pleistocenos, mesolíticos y neolíticos. La secuencia estratigráfica está compuesta en ambos casos
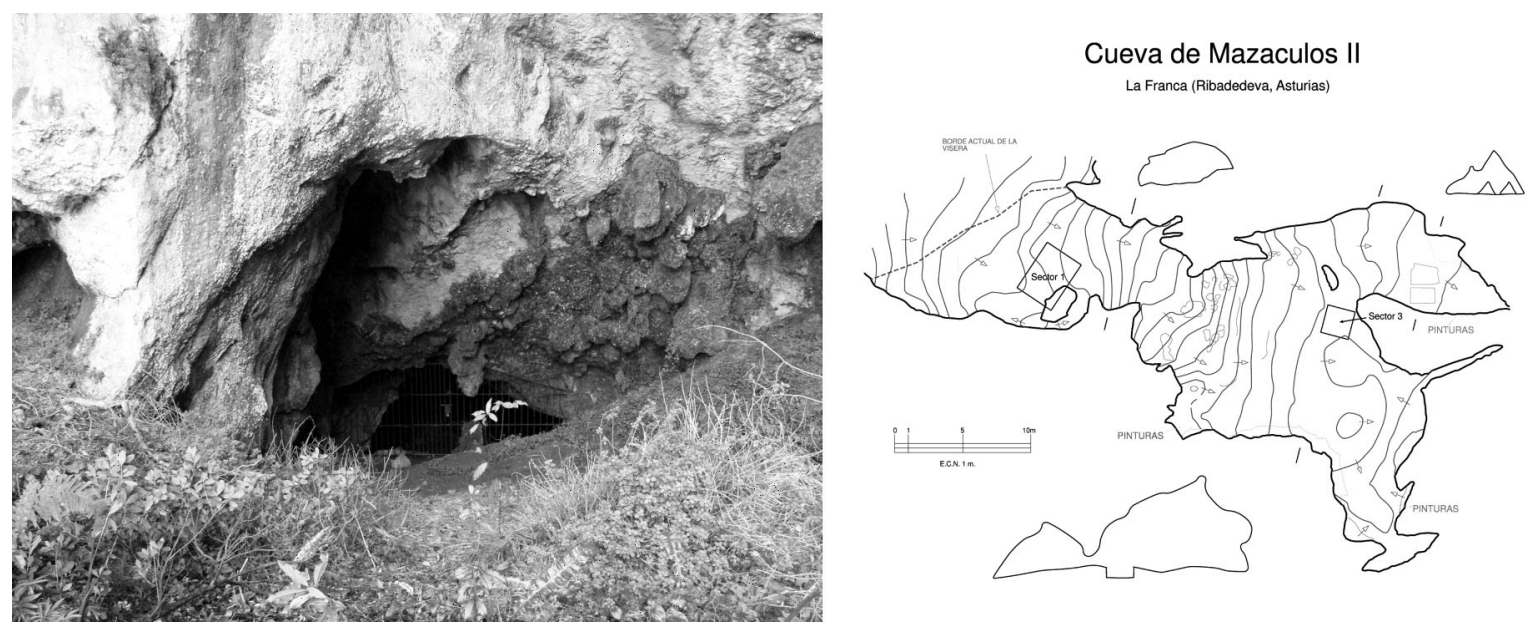

Lám. I. Vista exterior del Abrigo de Mazaculos II y topografía (fotografía: I. Gutiérrez Zugasti). 


\begin{tabular}{|c|c|c|c|c|c|c|}
\hline Zona & Nivel & Subnivel & Atrib. cultural & Nivel datado & $\mathrm{C}-14$ & Ref. Lab \\
\hline $\begin{array}{l}\text { Sector 3: } \\
\text { Interior }\end{array}$ & $\begin{array}{l}\text { A1 } \\
\text { A2 } \\
\text { A3 } \\
\text { A4 }\end{array}$ & $\begin{array}{c}\text { A2, A2 base, } \\
\text { A2 fondo } \\
- \\
-\end{array}$ & $\begin{array}{l}\text { Revuelto } \\
\text { Neolítico } \\
\text { Asturiense } \\
\text { Pleistoceno } \\
\text { indet. }\end{array}$ & $\begin{array}{l}\text { A2 } \\
\text { A3 }\end{array}$ & $\begin{array}{l}5050 \pm 120 \\
7030 \pm 120\end{array}$ & $\begin{array}{l}\text { GaK-15221 } \\
\text { GaK-15222 }\end{array}$ \\
\hline $\begin{array}{l}\text { Sector 1: } \\
\text { Abrigo } \\
\text { Exterior }\end{array}$ & $\begin{array}{l}3 \\
4\end{array}$ & $\begin{array}{c}1.1,1.2,1.3 \\
2.1,2.2 \\
3.1,3.2,3.3 \\
\text { (sup. } 1, \text { sup. } 2 \text {, } \\
\text { sup. } 3 \text {, sup. } 3 \\
\text { arcilla) }\end{array}$ & $\begin{array}{l}\text { Asturiense } \\
\text { Pleno } \\
\text { Asturiense } \\
\text { Inicial } \\
\text { Pleistoceno } \\
\text { indet. }\end{array}$ & 1.1 & $\begin{array}{l}7280 \pm 220 \\
9290 \pm 440\end{array}$ & $\begin{array}{l}\text { GaK-8162 } \\
\text { GaK-6884 }\end{array}$ \\
\hline
\end{tabular}

Tab. 1. Cronología de la Cueva de Mazaculos II. Todas las muestras fueron datadas en carbón vegetal en años convencionales de C-14, vida media Libby.

por cuatro niveles, que han proporcionado una serie coherente de dataciones absolutas que permiten establecer una adecuada secuencia de ocupación (Tab. 1) (González Morales 1995b).

La datación del nivel 3.3 constituye la fecha más antigua para un conchero asturiense, superando a la obtenida en el nivel 1.3 del Abrigo del Perro (9260 \pm 60 BP) (González Morales y Díaz 1991/92).

\subsection{El registro óseo}

Entre las distintas posibilidades ofrecidas por el registro arqueológico de la cueva de Mazaculos II, el presente trabajo se centra en el análisis arqueozoológico del conjunto fósil de macromamíferos del conchero exterior (niveles 1 a 3 ) y del estrato neolítico de la galería interior (A2). El nivel A1 fue desechado por tratarse de un revuelto, con abundante mezcla reciente. El nivel A3 sufrió alteraciones posdepósito de lavado del estrato, que también afectaron, pero en menor medida, a la unidad inmediatamente superior. Por este motivo se decidió no considerar en este estudio los materiales de A3. Los resultados de los niveles pleistocenos (con escasos restos) no se han incluido en este estudio, fundamentalmente orientado a la interpretación de los modos de vida mesolíticos y neolíticos. Igualmente, los restos de micromamíferos, aves y peces no se incluyen en este trabajo ya que su estudio se encuentra aún en curso. El nivel 3 contaba con un listado de restos identificados desde el punto de vista taxonómico realizado por Jesús Altuna en 1987; ahora se ha revisado y completado con el análisis tafonómico para equipararlo al del resto de niveles. Los 4 niveles examinados, con sus correspondientes subniveles $(1.1,1.2,1.3,2.1,2.2,3$ en la parte exterior), se han agrupado para su posterior interpretación en las siguientes divisiones cronológicas: (1) Asturiense inicial: nivel 3, (2) Asturiense pleno: niveles 1 y 2, (3) Neolítico: nivel A2 en su conjunto.

Los estratos analizados presentan gran abundancia de restos óseos en comparación con otros concheros de este período, lo que viene a confirmar la importancia del yacimiento. Se han inventariado un total de 1.825 restos con un peso de $10,83 \mathrm{~kg}$. Cada uno de los restos óseos fue observado bajo lupa binocular en busca de alteraciones tafonómicas visibles. Los restos identificables fueron comparados con atlas anatómicos (Pales y Lambert 1972; Schmid 1972) y diversas colecciones de referencia (1) para determinar el taxón y la parte esquelética de procedencia. Los restos que no pudieron identificarse taxonómicamente pero si anatómicamente fueron agrupados en la categoría de "Mamíferos" (grandes, medianos y pequeños). Así dentro de Mamífero Grande se han agrupado los posibles restos de equino y bovino, mientras que dentro de la categoría de

(1) Las colecciones consultadas son las depositadas en la Universidad de Cantabria, la Sociedad de Ciencias Aranzadi y la Universidad Autónoma de Madrid. 
Mamífero Medio se han englobado los siguientes animales: ciervo, suido, cabra/oveja, rebeco, corzo y perro/lobo. Por último, los restos de talla de Mamífero Pequeño agrupan a animales de la talla de lepóridos, insectívoros y pequeños carnívoros como la nutria, el zorro, el tejón. De esta forma, un $61,5 \%$ y $53,2 \%$ del total han sido identificados anatómica y taxonómicamente respectivamente. Si se incluye la categoría genérica de "Mamíferos", el porcentaje de restos identificados taxonómicamente se eleva a 89,4 \%. En la tabla 2 se presentan la distribución por especies y niveles del material estudiado.

Desde un punto de vista tafonómico, la ocupación antrópica de la cueva ha quedado evidenciada tanto por las marcas de carnicería identificadas en los huesos, como por el patrón de fracturación ósea, las huellas de termoalteraciones y la representación anatómica de los animales cazados. Las marcas de corte aparecen en, aproximadamente, el $17,1 \%$ de los restos identificados taxonómicamente, mientras que este porcentaje alcanza el $25,9 \%$ en el caso de fracturas en fresco para el aprovechamiento medular. El grado de fracturación de la muestra, calculado como cociente entre el peso total y el NR, es idéntico en los niveles mesolíticos $(8,6)$, mientras que en el neolítico es algo menor $(9,4)$. Un total de cinco remontajes y cinco rearticulaciones han podido realizarse en el conjunto total. Entre los primeros, algunos de ellos (falange, metatarso y sacro) corresponden a elementos fracturados para su aprovechamiento medular y de grasa mientras que, entre los elementos rearticulados, la dispersión espacial de los restos indica la escasa remoción del depósito.

Además, de las alteraciones producidas durante el procesado de las carcasas, se han podido apreciar otras alteraciones tafonómicas. Entre las modificaciones bioestratinómicas cabe señalar la actividad de carnívoros $(1,1 \%$ del total) y meteorización $(1,7 \%$ del total $)$, mientras que entre las diagenéticas, se han observado mayor presencia de vermiculaciones y concreciones en los restos de la zona exterior que de la interior. En cambio, los huesos teñidos por minerales como hierro y manganeso son más frecuentes en la zona interior de la cueva. Por último, otro tipo de alteraciones como huellas de pisoteo han dejado en la superficie de algunos huesos perforaciones y aplastamientos, mientras que la disolución ha deslavado algunos restos. Estas dos últimas alteraciones se observan en ambas zonas.

\subsection{Cuantificación}

En lo que respecta a la cuantificación del conjunto, los huesos identificables fueron evaluados mediante los siguientes índices: Número de Restos (NR), Número Mínimo de Individuos (MNI) (Klein y Cruz Uribe 1984), Número Mínimo de Elementos (NME) y Unidades Mínimas Animales (MAU) (Binford 1978).

Siguiendo la tendencia actual, el MNE no fue estimado únicamente a partir de las epífisis sino que se contabilizaron también las diáfisis (Bartram y Marean 1999; Cleghorn y Marean 2004; Marean et al. 2001, 2004; Pickering et al. 2003). De hecho, la exclusión de las diáfisis de huesos largos en el análisis generalmente se considera una práctica poco fiable, pues puede crear una subestimación de los huesos largos, desvirtuando, por tanto, la interpretación de los perfiles esqueléticos (ver estado de la cuestión en Marín, en prensa a). Así pues, cada uno de los elementos anatómicos, incluyendo las diáfisis, fue dividido en diversas secciones o zonas diagnósticas con independencia de las áreas de la inserción muscular. Cada una de estas secciones cuenta con propiedades morfológicas particulares que posibilitan su identificación o, en el caso de las diáfisis medias, con la porción de circunferencia que supone. Los restos óseos fueron asignados a una o varias secciones según su contribución al elemento completo. Finalmente, el NME final de cada elemento, por especies, se obtiene como el mayor valor resultante de la suma de cada sección. En la tabla 3 se recoge el NME por partes anatómicas de los ungulados de los niveles 3 y A2, que son los que disponen de un registro suficientemente extenso como para permitir un estudio estadísticamente representativo de los perfiles esqueléticos.

\subsection{Edad y sexo}

En los casos en los que fue posible, se estimó la edad del individuo a partir tanto de la erupción y el desgaste dental como del grado de fusión de las epífisis óseas. Para el caso de la fusión ósea de bóvidos, ovicaprinos, y cánidos se utilizaron los trabajos de Silver (1980), para suidos los datos de Bull y Payne (1982), mientras que para el corzo se emplearon las determinaciones de Tomé y Vigne (2003). En el caso del ciervo, el principal taxón identificado, la erupción y desgaste dental 


\begin{tabular}{|c|c|c|c|c|c|c|}
\hline & Nivel & Especie & NR & $P(g)$ & NME & NMI \\
\hline $\begin{array}{l}\text { Asturiense } \\
\text { Inicial }\end{array}$ & 3 & $\begin{array}{c}\text { Bos primigenius } \\
\text { Bos sp. } \\
\text { Cervus elaphus } \\
\text { Capreolus capreolus } \\
\text { Rupicapra rupicapra } \\
\text { Sus scrofa } \\
\text { Erinaceus europeus } \\
\text { Meles meles } \\
\text { Mamífero medio } \\
\text { No identificable } \\
\text { Total }\end{array}$ & $\begin{array}{r}12 \\
64 \\
486 \\
75 \\
1 \\
48 \\
1 \\
2 \\
393 \\
300 \\
\mathbf{1 . 3 8 4}\end{array}$ & $\begin{array}{r}744,3 \\
1.272,3 \\
3.218 \\
415,8 \\
5,7 \\
284,8 \\
0,9 \\
8,1 \\
723,4 \\
190 \\
\mathbf{6 . 8 9 9 , 1}\end{array}$ & $\begin{array}{r}12 \\
33 \\
199 \\
46 \\
1 \\
36 \\
1 \\
1 \\
\\
\\
331\end{array}$ & $\begin{array}{r}4 \\
3 \\
12 \\
5 \\
1 \\
4 \\
1 \\
1 \\
\\
\\
33\end{array}$ \\
\hline $\begin{array}{l}\text { Asturiense } \\
\text { Pleno }\end{array}$ & $\begin{array}{l}2.1 \\
2.2\end{array}$ & $\begin{array}{c}\text { Cervus elaphus } \\
\text { No identificable } \\
\text { Total } \\
\text { Cervus elaphus } \\
\text { Capreolus capreolus } \\
\text { Sus scrofa } \\
\text { Mamífero medio } \\
\text { Total } \\
\text { Cervus elaphus } \\
\text { Capreolus capreolus } \\
\text { Mamífero medio } \\
\text { Mamífero pequeño } \\
\text { Total } \\
\text { Cervus elaphus } \\
\text { Capreolus capreolus } \\
\text { Sus scrofa } \\
\text { Mamífero medio } \\
\text { No identificable } \\
\text { Total } \\
\text { Cervus elaphus } \\
\text { Sus scrofa } \\
\text { Mamífero medio } \\
\text { Total } \\
\text { Cervus elaphus } \\
\text { Total }\end{array}$ & $\begin{array}{r}12 \\
1 \\
\mathbf{1 3} \\
15 \\
2 \\
4 \\
5 \\
\mathbf{2 6} \\
2 \\
1 \\
1 \\
1 \\
\mathbf{5} \\
1 \\
1 \\
2 \\
7 \\
1 \\
\mathbf{1 2} \\
2 \\
1 \\
1 \\
\mathbf{4} \\
1 \\
\mathbf{1}\end{array}$ & $\begin{array}{r}80,5 \\
0,6 \\
\mathbf{8 1 , 1} \\
146,3 \\
3,9 \\
76,8 \\
13,4 \\
\mathbf{2 4 0 , 4} \\
2,3 \\
5,8 \\
3,7 \\
0,3 \\
\mathbf{1 2 , 1} \\
9,3 \\
16,8 \\
7,1 \\
10,8 \\
2,6 \\
\mathbf{4 6 , 6} \\
8,3 \\
1,4 \\
0,9 \\
\mathbf{1 0 , 6} \\
17,9 \\
\mathbf{1 7 , 9}\end{array}$ & $\begin{array}{r}10 \\
\\
\mathbf{1 0} \\
15 \\
2 \\
4 \\
\\
\mathbf{2 1} \\
2 \\
1\end{array}$ & $\begin{array}{l}\mathbf{2} \\
2 \\
1 \\
1 \\
4 \\
2 \\
1\end{array}$ \\
\hline Neolítico & A2 & $\begin{array}{c}\text { Bos primigenius } \\
\text { Bos sp. } \\
\text { Bos taurus } \\
\text { Cervus elaphus } \\
\text { Capreolus capreolus } \\
\text { Rupicapra rupicapra } \\
\text { Sus sp. } \\
\text { Sus scrofa } \\
\text { Ovicaprinos } \\
\text { Ovicaprinos domest } \\
\text { Canis lupus } \\
\text { Canis sp. } \\
\text { Vulpes vulpes } \\
\text { Ursus sp. } \\
\text { Lutra lutra } \\
\text { Mamífero medio } \\
\text { Mamífero pequeño } \\
\text { No identificable } \\
\text { Total }\end{array}$ & $\begin{array}{r}1 \\
15 \\
2 \\
245 \\
40 \\
5 \\
30 \\
3 \\
22 \\
4 \\
1 \\
1 \\
1 \\
2 \\
1 \\
4 \\
3 \\
1 \\
\mathbf{3 8 1}\end{array}$ & $\begin{array}{r}72,1 \\
329,4 \\
11,6 \\
2.564 \\
107,2 \\
26,5 \\
216,7 \\
89,4 \\
59,2 \\
20,1 \\
0,9 \\
6,5 \\
7,8 \\
7,4 \\
0,6 \\
26,3 \\
0,9 \\
1,4 \\
\mathbf{3 . 5 4 8}\end{array}$ & $\begin{array}{r}1 \\
11 \\
2 \\
111 \\
30 \\
5 \\
27 \\
3 \\
17 \\
3 \\
1 \\
1 \\
1 \\
2 \\
1\end{array}$ & $\begin{array}{l}1 \\
1 \\
1 \\
5 \\
2 \\
1 \\
2 \\
2 \\
4 \\
2 \\
1 \\
1 \\
1 \\
1 \\
1\end{array}$ \\
\hline & & Total muestra & 1.825 & $10.837,9$ & 588 & 72 \\
\hline
\end{tabular}

Tab. 2. Número de Restos, Peso, Número Mínimo de Elementos y Número Mínimo de Individuos del conjunto de niveles analizados de Mazaculos II. Bos primigenius: uro; Bos taurus: vaca; Cervus elaphus: ciervo; Capreolus capreolus: corzo; Rupicapra rupicapra: rebeco; Sus scrofa: jabalí; Meles meles: tejón; Canis lupus: lobo; Vulpes vulpes: zorro; Lutra lutra: nutria; Ursus sp.: oso; Erinaceus europeus: erizo. 


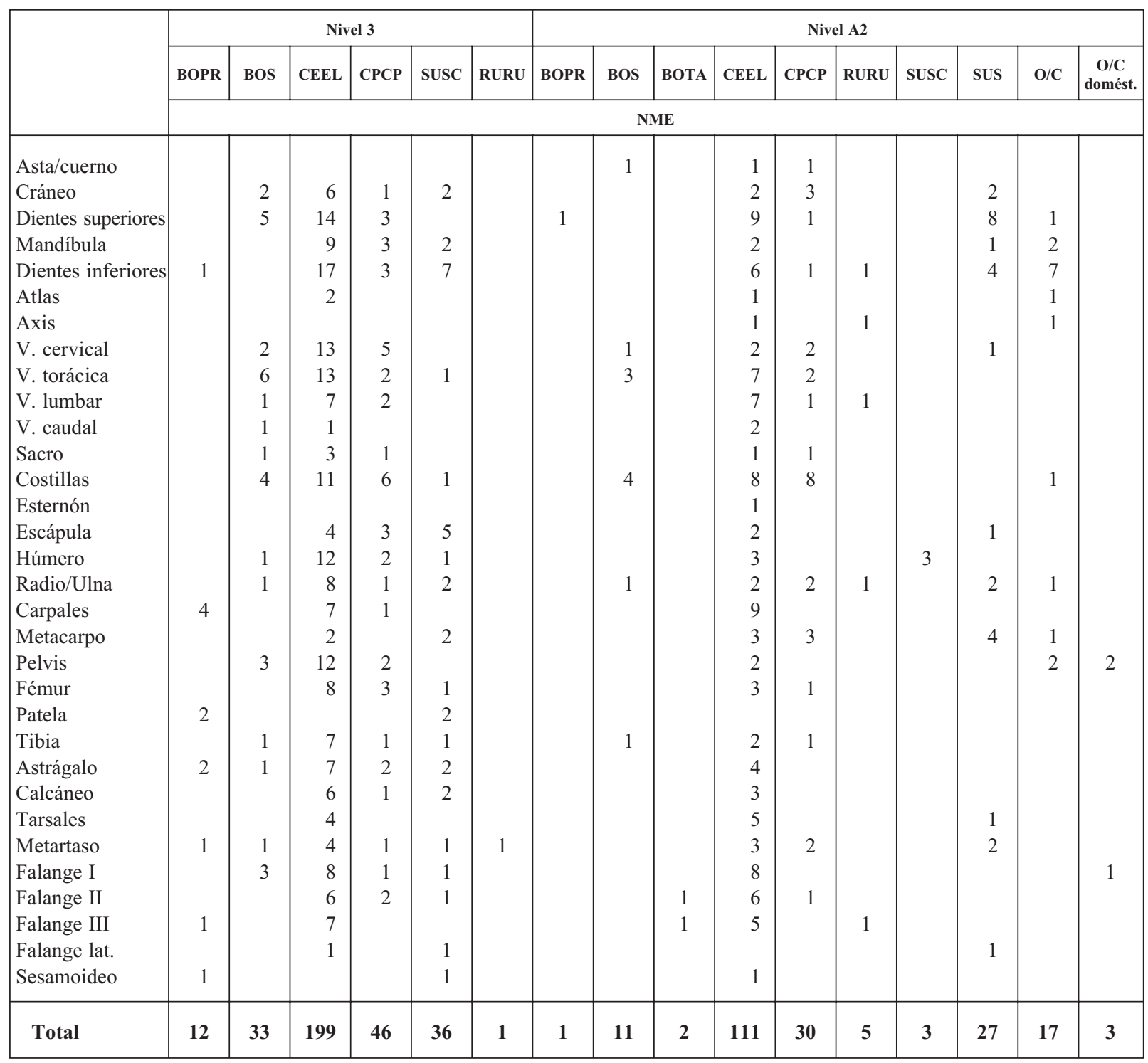

Tab. 3. Número Mínimo de Elementos (NME) de Ungulados de los niveles A2 y 3 de Mazaculos II. BOPR: Bos primigenius; BOTA: Bos taurus, BOS: Bos/Bison; CEEL: Cervus elaphus CPCP: Capreolus capreolus, RURU: Rupicapra rupicapra, SUSC: Sus scrofa SUS: Sus sp., O/C: Ovicaprinos; O/C domést.: Ovicaprinos domésticos; V. Vértebra.

fue valorado mediante el estudio de Azorit et al. (2002), mientras que la relación de la fusión ósea con la edad siguió lo establecido por Purdue (1983) y Mariezkurrena (1983).

En la definición de los datos de estacionalidad, se han considerado las siguientes épocas de nacimiento, aproximadas, para las distintas especies identificadas en el yacimiento: bovinos entre abril y mayo (Arnold y Greenfield 2006), ciervo y corzo entre mayo y junio (Carranza 2004; Mateos-Quesada 2005), rebeco entre abril y mayo (Pérez Barbería y García-González 2004) y suidos entre febrero y abril (Fernández-Llario 2006).

\subsection{Osteometría}

En los casos en los que la fracturación del resto no lo ha impedido, y siempre y cuando el resto no haya sufrido alteración natural o antrópica que haya modificado su dimensión original, se ha procedido a tomar sus dimensiones. Los criterios 
y codificación aquí empleados han sido tomados de Von den Driesch (1976). A continuación, en la tabla 4 se indican las abreviaturas utilizadas en el estudio osteométrico.

\subsection{Análisis paleoeconómico}

La cuantificación del grado de homogeneidad y diversidad de la muestra taxonómica, relacionada con la amplitud de la dieta y el grado de aprovechamiento antrópico del medio, se ha efectuado mediante la obtención del Inverso del Índice de Simpson (1949) cuyo valor aumenta con el número de especies consumidas y con la uniformidad en sus capturas. Este índice se calcula mediante la siguiente fórmula:

$$
I s p=\frac{1}{\sum_{i=1}^{k} p_{i}^{2}}
$$

donde $p_{i}$ es la frecuencia de cada especie sobre el total de la muestra y $k$ es el número de especies cazadas. Su valor oscila entre 1 y el número de especies explotadas.

A su vez, la importancia de cada especie en la subsistencia de los grupos humanos se ha evaluado desde dos puntos de vista: 1) a partir de la representatividad del número de restos en la muestra (como hasta el momento se ha venido realizando en los estudios arqueozoológicos) y 2) a partir de la estimación aproximada de biomasa aportada por cada taxón recuperado en el yacimiento. Ambas metodologías permiten acercarse al conjunto desde puntos de vista diferentes, si bien en ocasiones ofrecen resultados análogos. Para el cálculo de la biomasa aportada por cada animal se ha utilizado los valores de carne aprovechable presentados por Freeman (1973: 22). En el caso de los individuos juveniles dicho porcentaje ha sido disminuido en un 33,3\% y para los individuos infantiles en un $66,7 \%$ (ver Tab. 5). El valor total estimado de biomasa de cada especie se ha obtenido tras multiplicar su NMI, según su edad, por el valor en carne correspondiente (Tab. 6). Este procedimiento asume como hipótesis simplificadora que, independientemente del tipo de transporte diferencial desarrollado, toda la carne aprovechable de las carcasas obtenidas ha sido consumida por el grupo humano, lo cual resulta razonable a efectos comparativos. Con esta última estimación se pretende establecer de for- ma aproximada el aporte energético que cada animal pudo representar en la dieta, obviando los problemas inherentes a la comparación del NR, procedimiento que, si bien básico, se encuentra altamente influenciado por el grado de fracturación y la conservación diferencial en cada yacimiento.

\begin{tabular}{|c|l|}
\hline Ap & Medida antero-posterior de la articulación pro- \\
& ximal \\
Atr & Anchura condilo \\
B & Anchura \\
BG & Anchura del proceso glenoideo \\
BT & Anchura de la superficie articular distal \\
Bd & Anchura máxima distal \\
BFd & Anchura de la superficie articular distal \\
BFp & Anchura de la superficie articular proximal \\
Bp & Anchura maxima proximal \\
BPC & Anchura entre los procesos anconeos \\
DC & Espesor del caput \\
DI & Anchura medial \\
DLS & Longitud diagonal de la suela \\
Dm & Anchura lateral \\
DPA & Medida antero-posterior del Proceso ancóneo \\
& (ulna) \\
GB & Anchura máxima \\
GL & Longitud máxima \\
GLl & Longitud máxima de la zona medial \\
GLm & Longitud máxima de la zona lateral \\
GLP & Medida antero-posterior del proceso glenoideo \\
L & Longitud \\
Ld & Longitud dorsal \\
LG & Longitud máxima de la cavidad glenoidea \\
LO & Longitud del olecranon \\
MBS & Anchura en la mitad de la suela \\
SD & Espesor distal \\
SDO & Espesor mínimo del olecranon \\
SLC & Longitud mínima del cuello escapular \\
W & Espesor máximo de la zona articular \\
\hline
\end{tabular}

Tab. 4. Abreviaturas utilizadas en el estudio osteométrico.

\begin{tabular}{|l|c|c|c|}
\hline Carne aprovechable (kg) & Infantil & Juvenil & Adulto \\
\hline Bos sp. & 133,2 & 266,8 & 400 \\
Cervus elaphus & 33,3 & 66,7 & 100 \\
Capreolus capreolus & 4,2 & 8,3 & 12,5 \\
Capra pyrenaica & 16,7 & 33,4 & 50 \\
Rupicapra rupicapra & 7,5 & 15 & 22,5 \\
Sus sp. & 40 & 80 & 120 \\
Ovis aries & 5 & 10 & 15 \\
\hline
\end{tabular}

Tab. 5. Valores de carne estimados (en $\mathrm{kg}$ ) para cada una de las especies identificadas en el yacimiento de Mazaculos II atendiendo a su edad. 


\begin{tabular}{|c|c|c|c|c|c|c|}
\hline NMI & Nivel & Especie & $\begin{array}{c}\text { Infan- } \\
\text { til }\end{array}$ & $\begin{array}{c}\text { Juve- } \\
\text { nil }\end{array}$ & $\begin{array}{c}\text { Adul- } \\
\text { to }\end{array}$ & Total \\
\hline $\begin{array}{c}\text { Asturiense } \\
\text { Inicial }\end{array}$ & 3 & $\begin{array}{c}\text { Bos sp. } \\
\text { Uro } \\
\text { Ciervo } \\
\text { Corzo } \\
\text { Jabalí } \\
\text { Rebeco }\end{array}$ & $\begin{array}{l}1 \\
1 \\
8 \\
2 \\
1\end{array}$ & $\begin{array}{l}2 \\
1 \\
2\end{array}$ & $\begin{array}{l}2 \\
2 \\
2 \\
2 \\
1 \\
1\end{array}$ & $\begin{array}{r}4 \\
3 \\
12 \\
5 \\
4 \\
1\end{array}$ \\
\hline $\begin{array}{l}\text { Asturiense } \\
\text { Pleno }\end{array}$ & $\begin{array}{c}1 \\
1.1 \\
1.2 \\
1.3 \\
2.1 \\
2.2\end{array}$ & $\begin{array}{c}\text { Ciervo } \\
\text { Ciervo } \\
\text { Corzo } \\
\text { Jabalí } \\
\text { Ciervo } \\
\text { Corzo } \\
\text { Ciervo } \\
\text { Corzo } \\
\text { Jabalí } \\
\text { Ciervo } \\
\text { Jabalí } \\
\text { Ciervo }\end{array}$ & 1 & $\begin{array}{l}1 \\
1\end{array}$ & $\begin{array}{l}1 \\
1 \\
1 \\
1 \\
1 \\
1\end{array}$ & $\begin{array}{l}2 \\
2 \\
1 \\
1 \\
2 \\
1 \\
1 \\
1 \\
1 \\
1 \\
1 \\
1\end{array}$ \\
\hline Neolítico & A2 & $\begin{array}{c}\text { Bos sp. } \\
\text { Uro } \\
\text { Vaca } \\
\text { Ciervo } \\
\text { Corzo } \\
\text { Rebeco } \\
\text { Sus sp. } \\
\text { Jabalí } \\
\text { O/C } \\
\text { O/C } \\
\text { doméstico }\end{array}$ & $\begin{array}{l}1 \\
1\end{array}$ & $\begin{array}{l}1 \\
1 \\
2\end{array}$ & $\begin{array}{l}1 \\
1 \\
3 \\
1 \\
1 \\
1 \\
2 \\
1\end{array}$ & $\begin{array}{l}1 \\
1 \\
1 \\
5 \\
2 \\
1 \\
2 \\
2 \\
4 \\
\\
2\end{array}$ \\
\hline
\end{tabular}

Tab. 6. NMI de las especies identificadas, en cada uno de los períodos, por edades. $\mathrm{O} / \mathrm{C}$ ovicaprinos.

\subsection{La representación esquelética y su relación con la funcionalidad}

Para investigar en mayor detalle la funcionalidad de la cueva y el tipo de comportamiento económico adoptado por sus moradores, se ha realizado un detallado estudio de los perfiles esqueléticos asociados a los dos niveles con mayor significación estadística: 3 (Asturiense inicial) y A2 (Neolítico). Entre las técnicas analíticas actualmente disponibles para ello, se han aplicado las siguientes: el método de los perfiles anatómicos regionales $(A R P)$ desarrollado por Stiner (1991, 1994, 2002) únicamente para ciervo al ser el taxón con mayor número de restos, las correlaciones bivariables con índices de utilidad Modified General Utility Index (MGUI) (Binford 1981) y densidad ósea (Lam et al. 1999) aplicadas para ciervo y corzo, la diferenciación de partes anatómicas de alta y baja supervivencia (2) (Marean y Cleghorn 2003) y el cálculo de índices de homogeneidad (Faith y Gordon 2007) y el software Analysis of Bone Counts by Maximum Likelihead (ABCml) (Rogers 2000a, 2000b), empleadas estas dos últimas, exclusivamente, en los restos de ciervo.

El método de Stiner $(1991,1994,2002)$ permite la comparación homogénea del grado de supervivencia de cada una de las nueve partes anatómicas (3) en las que la autora divide al animal. Este método está basado en la suposición de que cada parte esquelética tiene un similar grado de poder sobrevivir, teniendo en cuenta su densidad media a partir de los valores propuestos por Lyman (1994). En este método, los dientes son excluidos ya que su estructura densa podría sobrerepresentar su abundancia en el depósito (Stiner 1991).

La aplicación de las correlaciones bivariables entre el \% Minimal Animal Units (MAU) y MGUI (Binford 1978) y entre el \% MAU y la máxima densidad ósea permite identificar el efecto tanto del transporte diferencial como de la atrición en la frecuencia esquelética del registro óseo analizado. Para estimar la naturaleza cuantitativa de esta correlación se ha empleado el coeficiente de correlación de Spearman (1904). A su vez, se ha obtenido el grado de significación (p) para determinar si la correlación es o no estadísticamente representativa. En el caso de las correlaciones \% MAU y $M G U I$ se ha optado por incorporar todos los elementos al análisis, en el convencimiento de que es precisamente la ausencia de determinadas partes anatómicas derivada del comportamiento humano lo que se pretende conocer. Sin embargo, en la comparación con densidad, se han eliminado los elementos con nula representación, ya que su consideración puede alterar significativamente los resultados cuando no se está se-

(2) Los huesos de alta supervivencia incluyen todos los huesos largos, mandíbula y cráneo, mientras que los huesos de baja supervivencia son las vértebras, costillas, pelvis, escápula, carpales/tarsales y falanges.

(3) 1: Cuernos/Astas; 2: Cabeza (sin dientes); 3: Atlas, Axis y vértebras cervicales; 4 : Demás vértebras, costillas y pelvis; 5: Escápula y húmero; 6: Radio/Ulna, metacarpo y carpales; 7: Fémur; 8: Tibia, metatarso y tarsales; 9: Metápodos y falanges.

T. P., 66, N. ${ }^{\circ} 1$, enero-junio 2009, pp. 47-74, ISSN: 0082-5638 doi: $10.3989 /$ tp. 2009.09012 
guro de que hayan sido aportados al asentamiento (Marín en prensa a).

Ampliando las oportunidades ofrecidas por las correlaciones bivariables, Marean y Cleghorn (2003) plantean la separación del registro en huesos de alta supervivencia y huesos de baja supervivencia antes de efectuar la comparación estadística, de forma que se pueda soslayar los efectos de los procesos atricionales, que afectarían de manera homogénea a todos los elementos de cada grupo. Sobre los elementos del conjunto de alta supervivencia, Faith y Gordon (2007) proponen la estimación del Índice de Homogeneidad de Shannon (Shannon y Weaver 1949) como representativo del tipo de transporte diferencial: completo, de cantidad o de calidad.

Por último, Rogers (2000a) ha desarrollado el citado método $A B C m l$ basado en la aplicación del Principio de Máxima Verosimilitud, que estima la acumulación ósea más probables de ser aportada a un yacimiento por cada tipo de agente (grupos humanos actuando en campamento residencial o de matanza: $\alpha_{\mathrm{k}}$ ), el número total de animales transportados $(\kappa)$ y el grado de atrición $(\beta)$. La probabilidad de supervivencia, a falta de mejores estimaciones, se hace depender de la densidad ósea (Rogers 2000b). Un valor de $\beta=1$ implica que únicamente sobreviven la mitad de los huesos, mientras que para $\beta=2$, el porcentaje de supervivencia se reduce hasta el $25 \%$. La bondad del método para representar el modelo económico desarrollado se evalúa mediante un test de ajuste $\chi^{2}$.

\section{RESULTADOS}

\subsection{Economía de subsistencia}

El análisis arqueozoológico efectuado ha revelado la existencia de una estrategia de subsistencia que, en función del tipo de resultado interpretado: NR o biomasa, difiere sobre cuales fueron los animales más importantes en la dieta de estos grupos humanos en los períodos estudiados. En cualquier caso, la economía aquí identificada dista mucho de la dieta especializada propia de momentos plenos del Paleolítico Superior (Marín 2008b, 2009).

Si observamos en la figura 2 los resultados a partir del número de restos (NR), el ciervo es el taxón más importante en todos los períodos. En el Asturiense Inicial, éste es seguido de lejos, y a igual nivel por el corzo y los bovinos, e inmediatamente después por el jabalí. En el Asturiense Pleno, la diversidad de especies y cantidad de restos se reduce drásticamente aunque el ciervo sigue siendo el taxón más destacado. No obstante, en este momento el jabalí sustituye al corzo en importancia después del ciervo, al contrario de lo

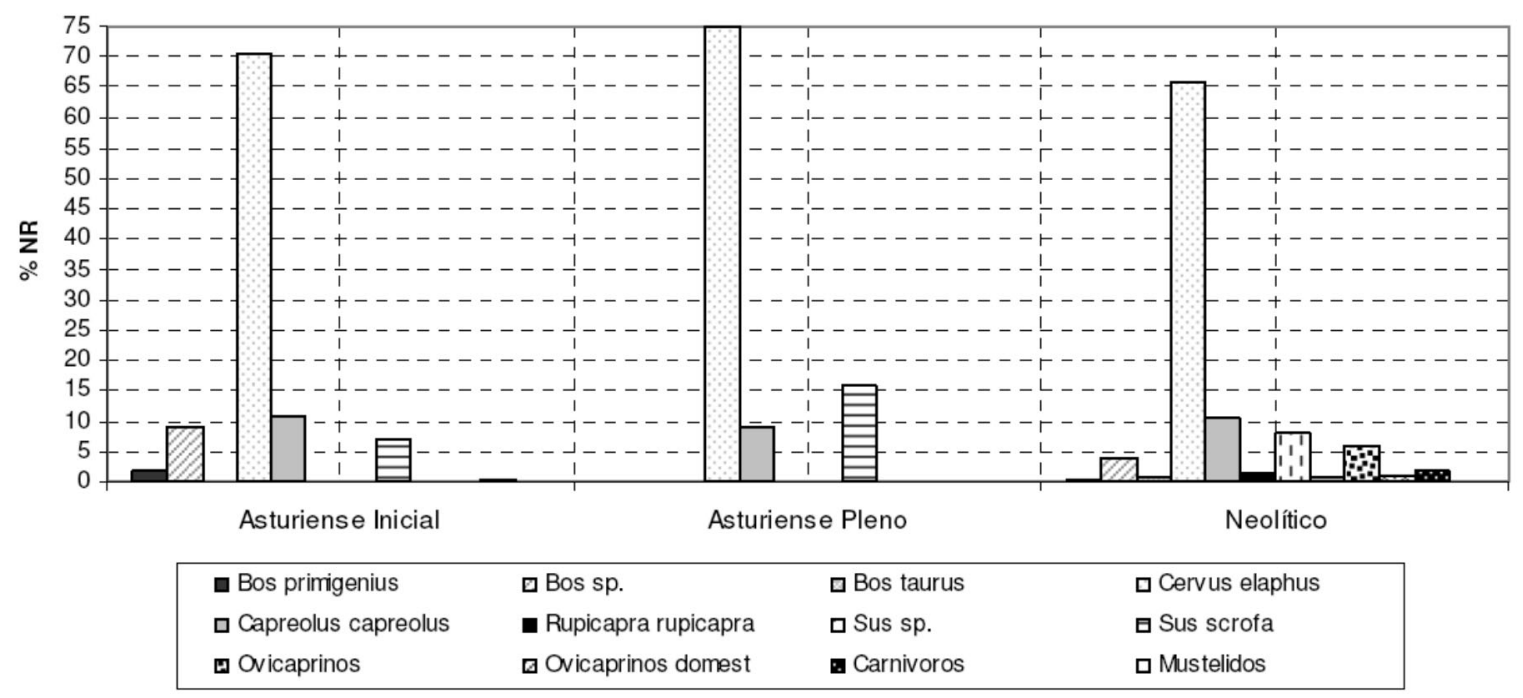

Fig. 2. Porcentajes del NR de los ungulados de los tres períodos de estudio de Mazaculos II. 


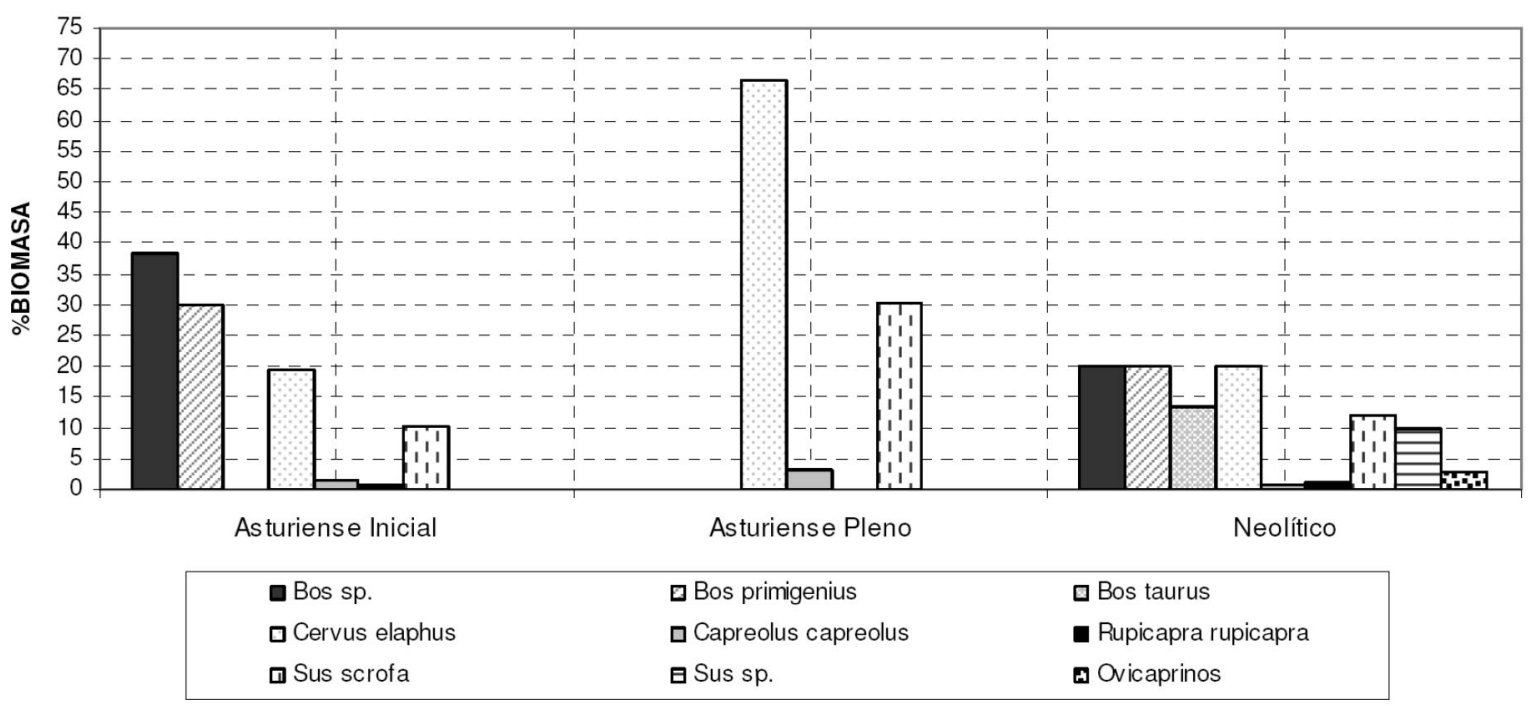

Fig. 3. Porcentajes de biomasa aportada por los ungulados consumidos en Mazaculos II.

que sucedía en el momento precedente. En el Neolítico, la amplitud de dieta vuelve a ser visible, sin embargo, el ciervo sigue mostrando niveles muy elevados con respecto a las otras especies, mientras que el corzo mantiene los niveles que mostraba en los períodos anteriores. Los suidos también tiene importancia similar a la de este último (si bien, no se ha podido identificar si los restos de suidos correspondían a jabalí o cerdo doméstico). La misma importancia muestran los bovinos y los ovicaprinos, aunque que con menor representación que los suidos. Por último, si bien aparecen representados animales domésticos, su importancia es todavía limitada.

Por otro lado, atendiendo al resultado que proporciona la biomasa como valor para aproximarse a la importancia de las especies consumidas en cada período, los resultados son sustancialmente diferentes a los obtenidos únicamente con el NR (Fig. 3). En el Asturiense inicial es destacable sobre todo la aportación de los bovinos a la dieta de los grupos humanos que ocuparon el yacimiento. En algunos casos se ha podido determinar que los individuos consumidos pertenecían a Bos primigenius, descartándose para el resto la existencia de bisonte, que, por otro lado, no ha sido identificado hasta el momento en ningún nivel Holoceno del área cantábrica (Mariezkurrena y Altuna 1995). El ciervo, si bien es el taxón más destacable en cuanto a NR, cuando se tiene en cuenta el aporte calórico asociado, queda relegado a un segundo término, en un nivel algo superior a la im- portancia del jabalí. La caza de corzo y del rebeco, a tenor, de su aportación, puede considerarse esporádica. A diferencia de la interpretación obtenida con el NR, estos resultados contrastan significativamente con la creencia clásica (Altuna 1995; Arias 1991; Clark 1976, 1995; Fano 2004, González Morales 1982; Straus 1992) que, basándose en los escasos restos cuantificados en otros yacimientos (excluyendo Urratxa III con evidentes problemas estratigráficos) otorgaba un papel dominante al ciervo. Así ocurre, por ejemplo, con los 74 restos de ciervo sobre 136 del total en $\mathrm{Cu}$ bio Redondo (Castaños 2001), los 17 sobre 34 de El Perro 1 (4), los 45 sobre 64 de Poza L'Egua (Arias et al. 2007b), los 113 sobre 122 de La Riera 29 (Altuna 1986) o los 27 sobre 48 de Balmori (Clark y Clark 1975) [ver, sin embargo, la predominancia de cabra en Los Canes (Castaños 2001)].

Siguiendo con la importancia calórica de cada animal, durante el Asturiense pleno, a juzgar por el número de restos recuperados, podría pensarse que el período de ocupación humana debió de ser mucho más esporádico, pero también hay que tener en cuenta la diferencia del tipo de depósito que se trata, por lo que no podemos considerarlo especialmente representativo. En cualquier caso, en este momento, al desaparecer el consumo de bovinos, es el ciervo el taxón más relevante en la

(4) Morales, A. y Moreno, R. 1998. El Abrigo de la Peña del Perro: el estudio óseo. Laboratorio de Arqueología, Universidad Autónoma de Madrid, informe interno 1998/8. 
dieta. Por último, durante el inicio del Neolítico, la estrategia de subsistencia vuelve al patrón observado en el Asturiense inicial, incorporándose además un nuevo factor: la domesticación, todavía con un rol muy secundario respecto de la caza.

En efecto, los restos encontrados han permitido reconocer evidencias de domesticación en ovicaprinos y bovinos. Así, se ha asignado una falange II y una falange III a un posible Bos taurus en función de su tamaño aunque no han podido ser medidos debido a pertenecer a un individuo con epífisis aún sin fusionar el primero y la fragmentación del segundo resto. En el caso de los ovicaprinos, dos pelvis y una falange I completamente fusionadas permiten su atribución inequívoca a animales ya domesticados (Lám. II). Comparando con otros restos neolíticos españoles (Castaños 2004), una de las pelvis, de reducido tamaño, podría corresponder a una oveja, si bien sus medidas son aproximadas, ya que el acetabulum aparece parcialmente fragmentado. Por su parte, el tipo de restos hallados de suidos no ha permitido su diferenciación en jabalí o cerdo doméstico. Las medidas de los huesos, que han podido ser medidos, se presentan a final del artículo.

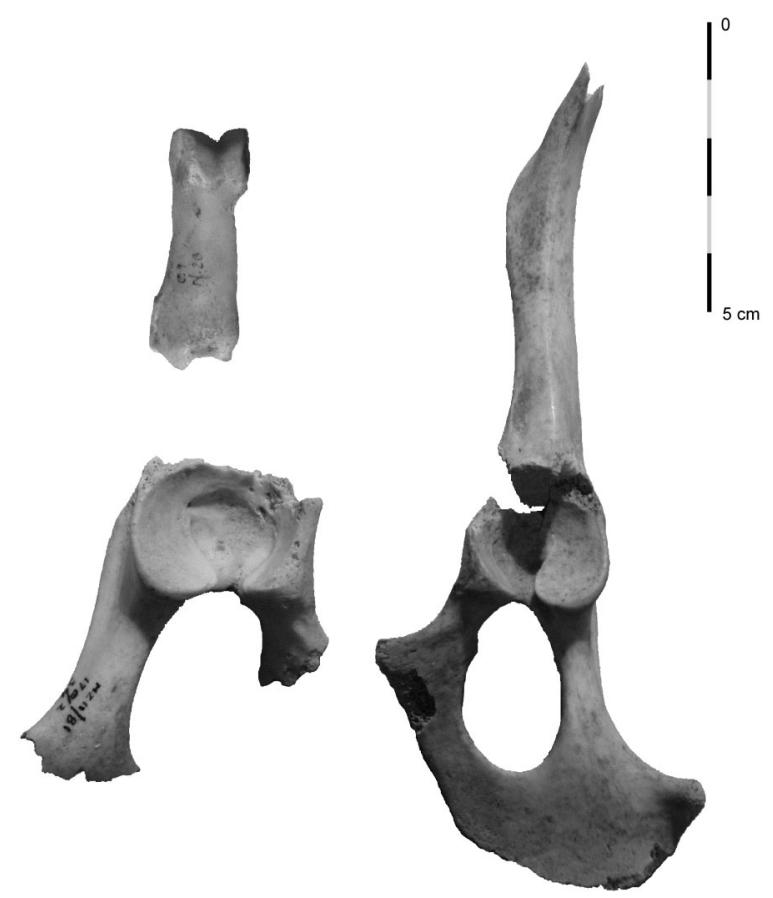

Lám. II. Pelvis y falange I de ovicaprinos del nivel A2 de Mazaculos II identificadas como animales domésticos.

\begin{tabular}{|c|c|c|c|c|c|}
\hline \multirow[b]{2}{*}{ Especie } & \multirow[b]{2}{*}{ Nivel } & \multicolumn{3}{|c|}{ Marcas de carnicería } & \multirow{2}{*}{$\begin{array}{c}\% \mathrm{NR} \\
\text { con } \\
\text { marcas } \\
\text { de } \\
\text { corte }\end{array}$} \\
\hline & & $\begin{array}{l}\text { Despe- } \\
\text { llejado }\end{array}$ & $\begin{array}{c}\text { Desar- } \\
\text { ticula- } \\
\text { ción }\end{array}$ & $\begin{array}{l}\text { Des- } \\
\text { car- } \\
\text { nado }\end{array}$ & \\
\hline Bos primigenius & 3 & & 7 & & 58,3 \\
\hline \multirow[t]{2}{*}{ Bos sp. } & 3 & & 2 & 5 & 10,9 \\
\hline & A2 & & 1 & 7 & 53,3 \\
\hline \multirow[t]{5}{*}{ Cervus elaphus } & 1 & & 1 & 2 & 25 \\
\hline & 1.1 & & & 2 & 13,3 \\
\hline & 1.3 & 1 & & & 100 \\
\hline & 3 & 8 & 38 & 43 & 18,3 \\
\hline & $\mathrm{A} 2$ & 8 & 28 & 45 & 33,1 \\
\hline \multirow{4}{*}{$\begin{array}{l}\text { Capreolus } \\
\text { capreolus }\end{array}$} & & & & & \\
\hline & 1.3 & & & 1 & 100 \\
\hline & 3 & & 14 & 16 & 40 \\
\hline & $\mathrm{A} 2$ & & 3 & 11 & 35 \\
\hline \multicolumn{6}{|l|}{ Rupicapra } \\
\hline rupicapra & $\mathrm{A} 2$ & & 3 & & 60 \\
\hline Sus sp. & $\mathrm{A} 2$ & & 10 & 4 & 46,7 \\
\hline \multirow[t]{3}{*}{ Sus scrofa } & 1.1 & & & 3 & 150 \\
\hline & 3 & 4 & 9 & & 27,1 \\
\hline & $\mathrm{A} 2$ & & 1 & & 33,3 \\
\hline Ovicaprinos & A2 & & 8 & 1 & 34,6 \\
\hline Meles meles & 3 & & & 1 & 50 \\
\hline Vulpes vulpes & A2 & & 1 & & 100 \\
\hline \multirow[t]{2}{*}{ Mamífero medio } & 1.1 & & & 1 & 20 \\
\hline & $\mathrm{A} 2$ & & & 2 & 50 \\
\hline \multirow{2}{*}{$\begin{array}{l}\text { Mamífero pequeño } \\
\text { (carnívoro) }\end{array}$} & & & & & \\
\hline & A2 & 1 & & & 33,3 \\
\hline
\end{tabular}

Tab. 7. Marcas de corte, por especies y niveles, relacionadas con las labores de carnicería en Mazaculos II.

Además del consumo de ungulados en la dieta como se ha podido observar a partir de las marcas de corte relacionadas con las labores de carnicería (Tab. 7) (Binford 1981; Pérez Ripoll 1992), se ha observado también el aprovechamiento alimenticio ocasional de carnívoros. Se trata de una ulna de tejón del nivel 3.3 en la que se han identificado marcas de descarnado (Lám. III), posiblemente cazado para conseguir su piel y, secundariamente, aprovechado como alimento. Lo mismo ocurre con una tibia de zorro que presenta trazas de desarticulación en la epífisis distal. Se ha identificado también un canino superior de nutria, un taxón poco común en la Prehistoria Cantábrica, hasta ahora sólo citado en el nivel Asturiense de Cueto de la Mina (Vega del Sella 1916) (si bien revisiones posteriores del material de ese yacimiento no pudieron comprobar su presencia (Castaños, 1982) y en Marizulo (Altuna 1972: 286). El consumo de carnívoros no es nuevo, ya ha sido documentado en otros yacimientos mesolíticos cantábricos como La Fragua (Marín 2004), en una tendencia que podría relacionarse con momentos de escasez. 


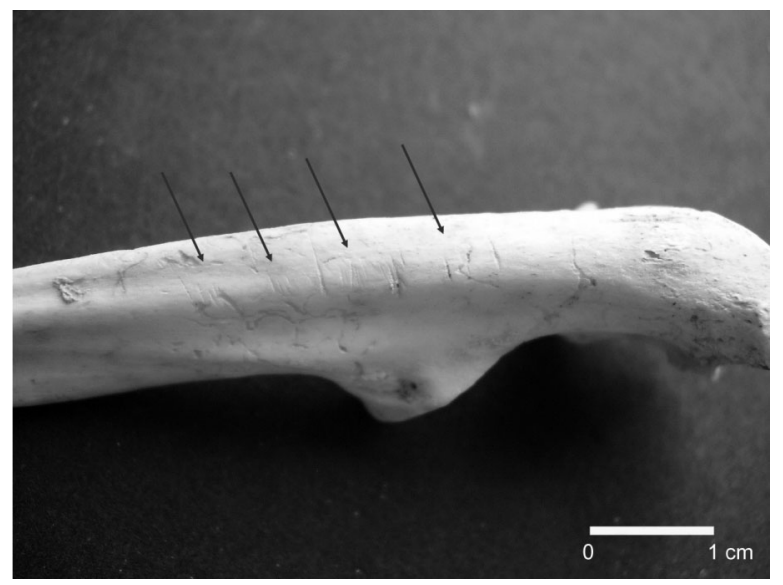

Lám. III. Trazas de descarnado en la zona caudal de una ulna de tejón realizadas para el aprovechamiento de su piel.

El estudio taxonómico muestra además, a diferencia de épocas anteriores y enlazando con la tendencia general del Holoceno (Aura et al. 1998; Clark, 1987; Straus 2004) una progresiva diversificación de la subsistencia. Esto se ha cuantificado en la figura 4, en la que se representa la evolución de la amplitud de dieta a partir de momentos magdalenienses. Así, se pasa de una economía dual centrada en el consumo de ciervo y/o cabra (Marín 2008b) a una creciente incorporación de otras especies como el corzo, el jabalí o los bovinos (González Sainz 1989; Straus 1992). Esto ha quedado evidenciado en el registro de Mazaculos II, si nos basamos en la metodología de estimación de la biomasa, en este caso, con una muestra suficientemente amplia como para ser considerada representativa.

Este incremento creciente de la amplitud de la dieta queda reforzado por el resto de evidencias fósiles del yacimiento, que incluyen, lógicamente, recursos malacológicos en elevada proporción, tanto del género Patella como Osilinus (5), pero también una amplia variedad de especies y tallas de peces (Roselló com. pers) lo que parece señalar una pesca poco selectiva.

Al mismo tiempo, se debe destacar la reducida aportación de mamíferos de roquedo a la dieta, lo

(5) Gutiérrez Zugasti, I. 2008. La explotación de moluscos y otros recursos litorales en la región cantábrica durante el Pleistoceno final y el Holoceno inicial. Tesis Doctoral inédita. Universidad de Cantabria. Santander.

Llera, E. y Ortea, J.A. Malacología de la cueva de Mazaculos. Un avance de resultados. Informe inédito. que, por otro lado, puede entenderse como la respuesta adaptativa de los grupos humanos a un entorno con máximas oportunidades de explotación de biotopos de llanuras aluviales y litorales en la desembocadura del río Cabra, en un escenario en el que la productividad energética evitaría, según criterios de optimización, la caza de animales de montaña (Marín 2008a).

\subsection{Estacionalidad}

Entre las evidencias faunísticas recuperadas en el yacimiento, la estacionalidad del yacimiento se ha podido establecer también a partir de los dientes de macromamíferos (ver Tab. 8). La amplitud de la época de explotación de la macrofauna está correlacionada con el número de indicadores validados (que se corresponde también con el número de restos de los distintos niveles). Esto podría interpretarse como que, a mayor duración de la ocupación, mayor acumulación de restos. Así, en el Asturiense inicial la ocupación de la cueva pudo producirse con una mayor intensidad entre los meses de marzo a octubre, reduciéndose en el Asturiense pleno a los meses finales de primavera, posiblemente debido a un uso más esporádico. En el Neolítico, la ocupación parece intensificarse, abarcando desde inicios de primavera a finales de verano. Respecto a la información isotópica $\mathrm{O}_{16 / 18}$ procedente de las conchas de varios moluscos (Osilinus) del nivel 3.3 (Asturiense antiguo) señala una recogida habitual en otoño e invierno con algún episodio primaveral, lo que, en conjunto, parece indicar un uso residencial y continuado del asentamiento en dicha época (González Morales 1995b).

En lo que respecta a la edad de muerte, se ha apreciado un significativo predominio de individuos infantiles (13 de 20) y juveniles (6 de 29) durante el Asturiense inicial, patrón que se invierte en épocas posteriores. Siguiendo una filosofía similar a la planteada por Stiner (1990) para la representación gráfica de los perfiles de edades de la fauna consumida, en la figura 5 se comparan los resultados obtenidos en Mazaculos con otros yacimientos cantábricos de cronología aziliense, mesolítica y neolítica con estudios arqueozoológicos completos. Destaca, como corresponde a una presión cinegética sostenible, la captura mayoritaria de individuos adultos en el Aziliense, con un ligero sesgo hacia estadios de desarrollo 


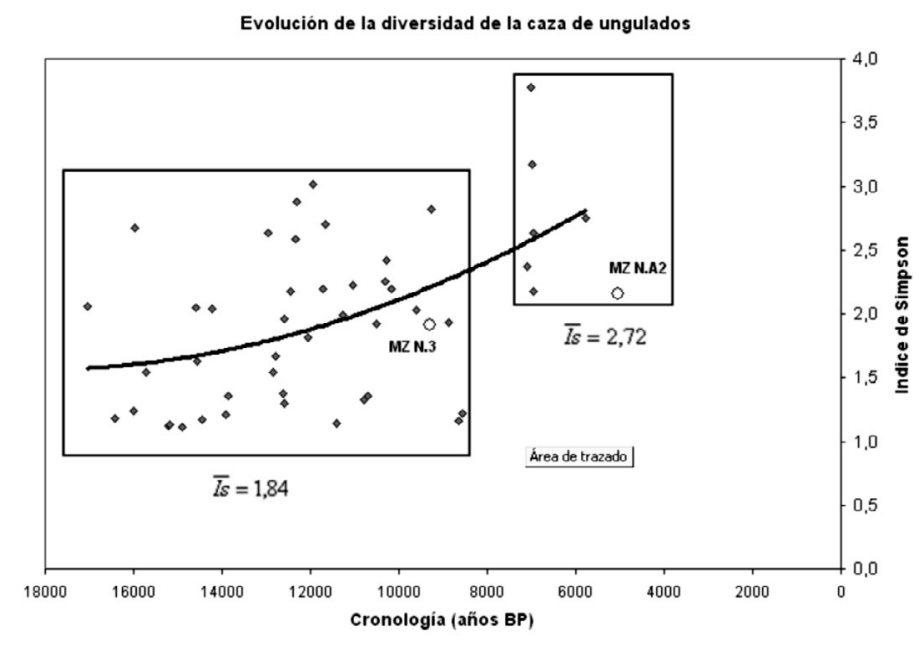

Fig. 4. Representación gráfica del Inverso del Índice de Simpson según el NR en base a los ungulados identificados desde el Magdaleniense Inicial hasta el Mesolítico en la Región Cantábrica. Se indican los niveles del Asturiense inicial y mesolítico de Mazaculos II.

biológico anteriores en momentos mesolíticos. En este contexto, la sorprendente diferencia del nivel 3 de Mazaculos podría ser interpretada como el resultado de una presión elevada sobre el medio, fruto de un desequilibrio alimenticio (Broughton 2002; Munro 1990, 2004; Speth 2006; Stiner 1994).

\subsection{Funcionalidad}

Con el propósito de conocer los efectos del transporte diferencial, así como de la atrición en las frecuencias de los perfiles esqueléticos, se presentan a continuación los resultados de los distintos métodos definidos. En primer lugar, los resultados del método de Stiner (1990), aplicados exclusivamente al ciervo, indican en el nivel 3 una sobrerrepresentación de la región 5 sobre el resto del esqueleto apendicular. Lo mismo puede decirse del cuello respecto a la zona axial. En el caso del nivel A2, es llamativa la elevada representación de restos de la cabeza y de la zona 5 con respecto a restos de extremidades (Fig. 6). Esto podría significar una preferencia hacia la contribución del esqueleto axial y

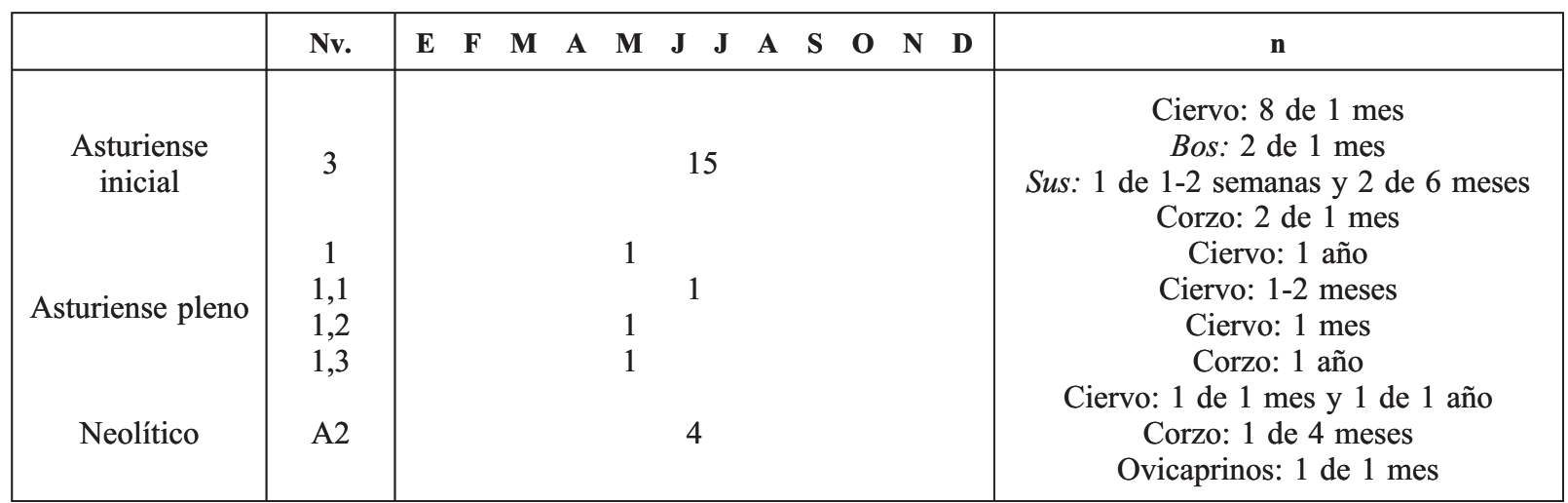

Tab. 8. Datos de estacionalidad en meses (columna central) en Mazaculos II según los restos de ungulados. Se indica el número de individuos (n) que han permitido conocer el momento de ocupación por niveles (Nv). 


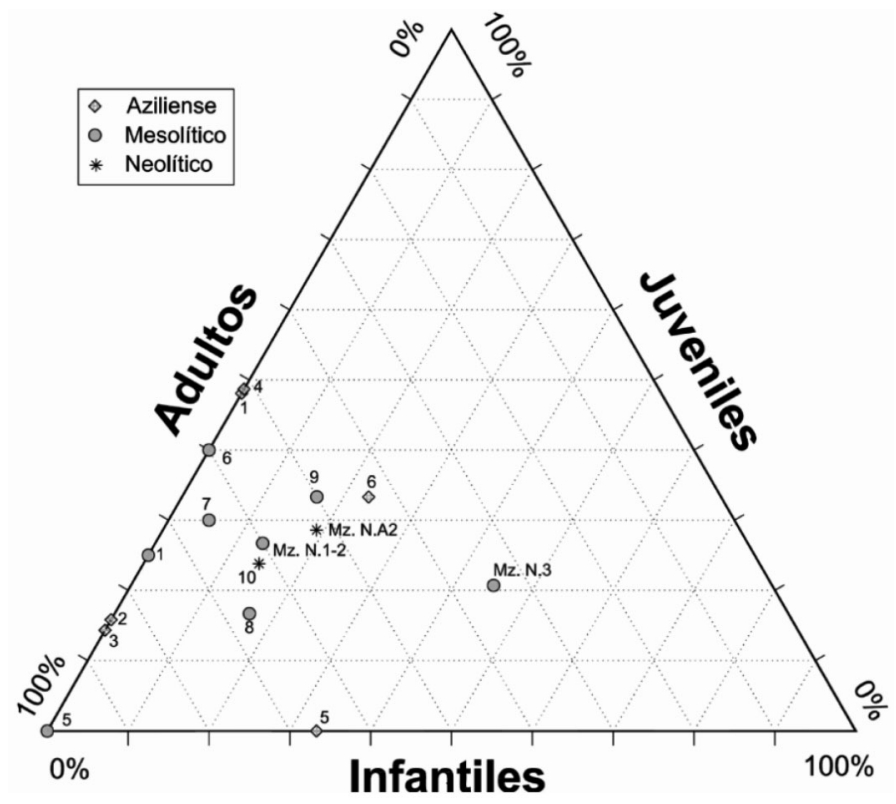

Fig. 5. Distribución de edades de ungulados en yacimientos cantábricos y su comparación con Mazaculos II. 1: La Riera (Altuna 1986), 2: La Paloma (Castaños 1980), 3: Cueto de la Mina (Castaños 1982), 4: Ekain (Altuna y Mariezkurrena 1984); 5: El Perro (Morales y Moreno 1998); 6: El Mirón (Altuna et al. 2004; Marín Arroyo nota 7); 7: La Fragua (Marín Arroyo 2004); 8: Kobeaga II (Castaños 1998/2000); 9: Cubio Redondo (Castaños 2001), 10: Herriko Barra (Mariezkurrena y Altuna 1995). Se aprecia la marcada presencia de restos infantiles en el Asturiense inicial de Mazaculos.

los miembros anteriores, si bien no es concluyente.

Para una mayor objetividad del análisis, la figura 7 muestra los resultados de las correlaciones bivariables para el ciervo y el corzo, los dos taxones más representados en el registro. Como puede apreciarse, si bien no existe una relación clara con la utilidad, sí es destacable el papel que la atrición ha desempeñado dentro del depósito, lo que, de hecho, ha sesgado la representación anatómica inicial, complicando la inferencia de hipótesis sobre el comportamiento humano.

Para mejorar la interpretación de la representación anatómica, se han efectuado también correlaciones entre el \% MAU y el MGUI de los elementos englobados en el grupo de alta supervivencia según Marean y Cleghorn (2003), sin obtener a su vez resultados satisfactorios. Por otro lado, se ha calculado también el Índice de Homogeneidad de Shannon para dichos elementos en el caso del ciervo, resultando un valor de 0,955 para un NME total de 56 en el nivel 3 y un valor de 0,990 para un NME total de 20 en el nivel A2. Según Faith y Gordon (2007), esto podría razonablemente interpretarse en términos de un transporte diferencial, al menos en el Asturiense inicial, lo que implicaría funcionalidades residenciales para el yacimiento.

Finalmente, se ha aplicado el método del $A B C m l$ (Rogers 2000) a la cuantificación de restos de ciervo (tantos en términos de NR como de NME) del los niveles 3 y A2. Para evitar un excesivo determinismo en la adopción de las hipótesis de partida que pudiera incorporar un sesgo en el estudio, se han establecido previamente dos estrategias de procesado en campo que difieren diametralmente en la forma en que las regiones anatómicas son descartadas en el lugar de matanza. En la primera, se tiende a desplazar el esqueleto axial al campamento base, en la idea de que el balance entre sus costes de descarnado y su utilidad justifica su aporte completo en detrimento del esqueleto apendicular del que únicamente se transportaría la carne. Esta estrategia coincide con las 

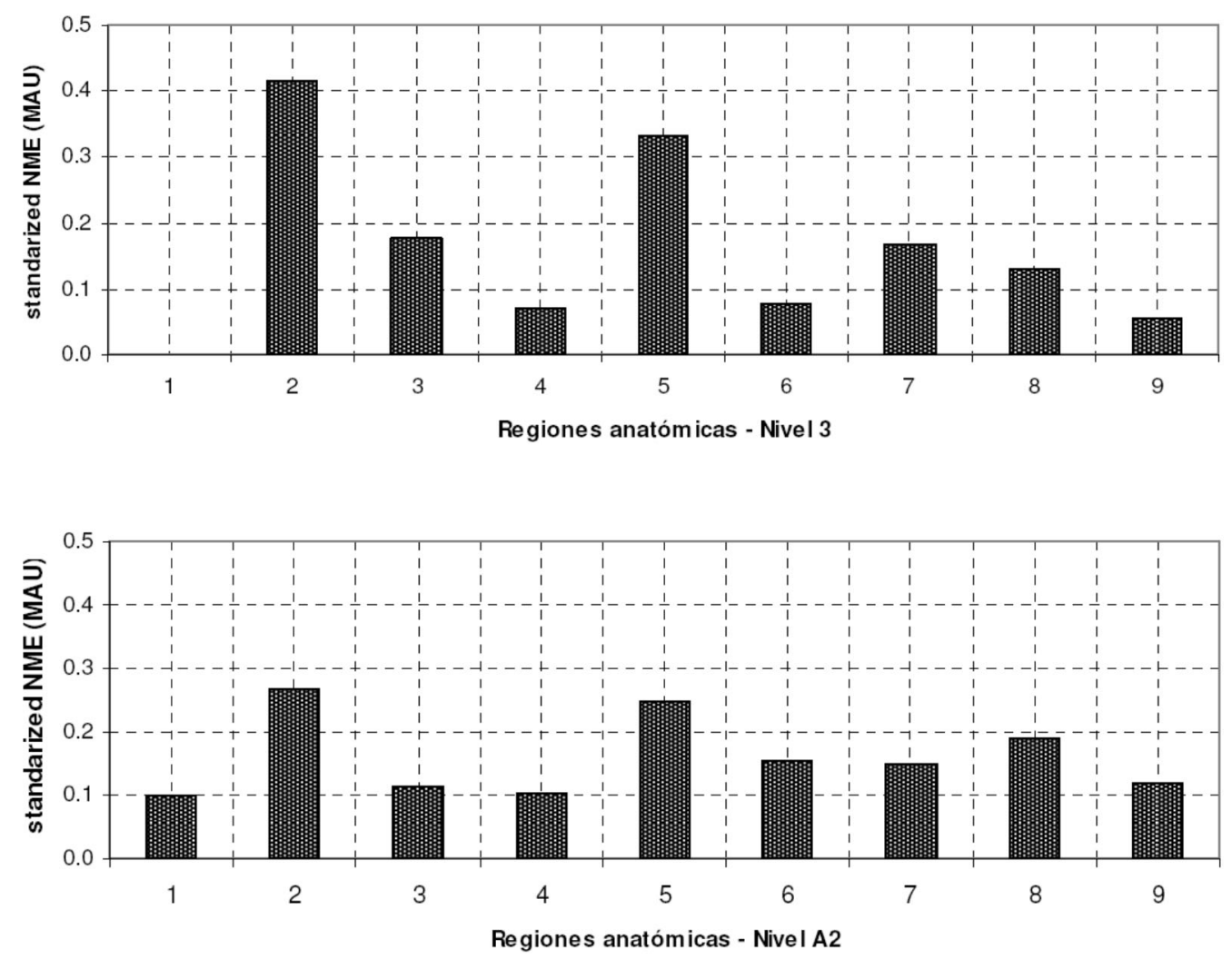

Fig. 6. Patrón de representación anatómica de Cervus elaphus del nivel 3 (arriba) y A2 (abajo) en Mazaculos II, basado en las nueve regiones anatómicas propuestas por Stiner (1991). 1: Asta/cuerno; 2: cabeza; 3: cuello; 4: esqueleto axial; 5: escápula/húmero; 6: radio/ulna, carpales y metacarpo; 7: fémur/rótula; 8: tibia, tarsales y metatarso; 9: falanges.

observaciones etnográficas realizadas entre grupos cazadores-recolectores actuales del continente africano en un escenario de imposibilidad de almacenamiento de las carcasas fuera del campamento base (Monahan 1998; O'Connell et al. 1988). En la segunda, siguiendo los postulados de Binford sobre índices de utilidad (1978), se prima el aporte del esqueleto apendicular, lo que implícitamente acepta una estrategia de transporte similar a las de los esquimales Nunamiut (Binford 1981), con un mínimo procesado en campo y la posibilidad de una contribución diferida en el tiempo.

Supuestas ambas estrategias, se han aplicado diferentes porcentajes de ocurrencia para cada tipo de contribución potencial: completa, completa sin cabeza y sólo esqueleto apendicular o axial, cubriendo todas la combinaciones posibles en intervalos de $10 \%$. Se ha querido evitar, de esta forma, restringir el número de configuraciones analizadas, ampliando el abanico de situaciones contempladas con el objetivo de identificar el mejor ajuste estadístico y, por tanto, la estrategia de procesado-transporte más plausible de entre todas las existentes. Tras este análisis, sin embargo, no se pudo establecer una estrategia única que consiguiera alcanzar valores del estadístico $\mathrm{x}^{2}$ por debajo de 0,59 tanto para NR como para NME, umbral mínimo establecido para asegurar una probabilidad de $90 \%$ de aceptar el modelo planteado. A pesar de esta dificultad, sí ha sido posible extraer ciertas conclusiones. Por un lado, se ha atestiguado la existencia de un atrición elevada en el depósito, con valores del parámetro $\beta$ que, en general superan la unidad, lo que supone la desaparición de, al menos, la mitad del registro original. En segundo lugar, la funcionalidad residencial, manifestada en los valores del parámetro $\alpha_{0}$, podría asociarse al yacimiento en el nivel 3, es decir, durante el Asturiense inicial en un $61 \%$ de los casos, mientras que dicho porcentaje se reduce al $44 \%$ para el nivel A2. Esto podría ser in- 


\section{Cervus elaphus Nivel 3}
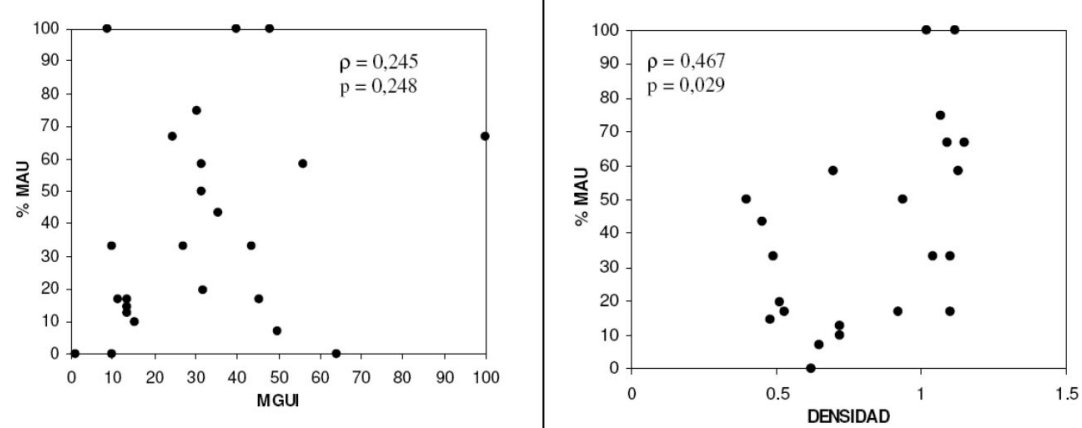

Capreolus capreolus Nivel 3
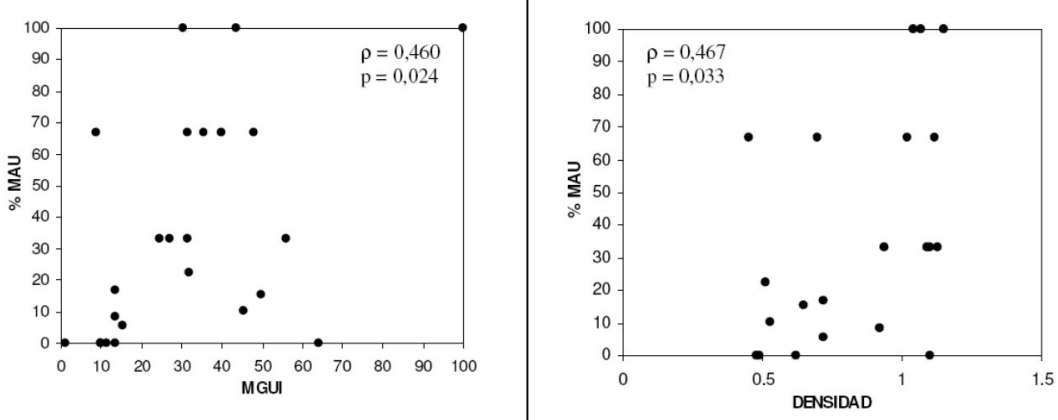

Cervus elaphus Nivel A2
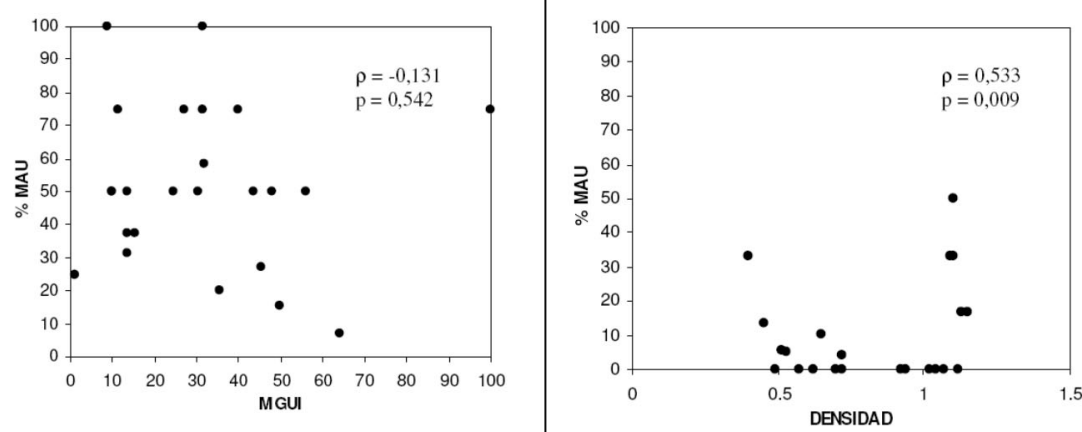

Capreolus capreolus Nivel A2
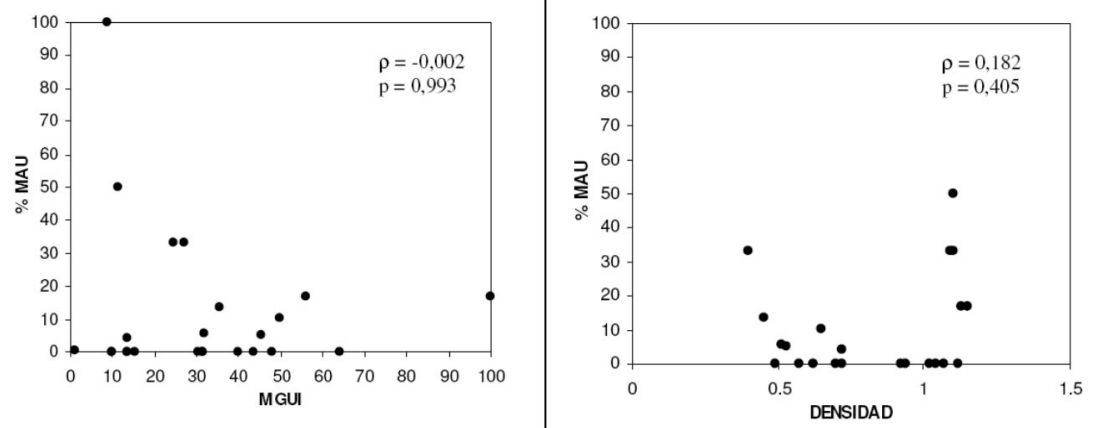

Fig. 7. Correlaciones bivariables entre la representación anatómica y la utilidad por un lado y con la densidad ósea por otro para ciervo y corzo en los niveles 3 y A2 de Mazaculos II. 
dicativo de un cambio paulatino en el uso de la cueva, con un empleo más logístico de la misma con la llegada del Neolítico.

En definitiva, parece confirmarse, a partir del registro faunístico, que el conchero asturiense de Mazaculos sería el resultado de un empleo residencial del abrigo, teoría ya postulada desde la excavación del yacimiento, tanto por la existencia de superficies de ocupación (González Morales 1982) como por los indicadores de estacionalidad.

\section{CONCLUSIONES Y DISCUSIÓN}

El yacimiento de Mazaculos II, cuya reexcavación se inició hace ya tres décadas posee unas cualidades idóneas para resolver algunos de los interrogantes planteados sobre el Mesolítico Cantábrico. Los estudios previos ya supusieron en su momento un impulso decisivo en el conocimiento de este período, al desterrar la idea de una posible funcionalidad secundaria de los concheros asturienses y al dotar a esta cultura de una identidad propia, no asociada a una hipotética explotación del litoral por parte de grupos azilienses asentados en los valles interiores (Fano 2004).

El trabajo que aquí se presenta es el resultado de la aplicación de las actuales técnicas de análisis estadístico empleadas en muestras arquezoológicas recuperadas en los niveles mesolíticos y neolíticos de este yacimiento, cuya importancia reside, no sólo en disponer de una secuencia estratigráfica que abarca toda la transición desde formaciones económicas cazadoras-recolectoras a otras productoras (González Morales 1982), sino en compartir, junto a Urratxa III y Herriko Barra en el Cantábrico Oriental, una de las colecciones más ricas de fauna del período, permitiendo, a diferencia del patrón típico de otros concheros mesolíticos, la obtención de conclusiones estadísticamente representativas. En este sentido, el estudio efectuado ha permitido establecer que: (1) basado en la representatividad del NR, el ciervo es el taxón más importante en los tres períodos analizados pero atendiendo al aporte cárnico de cada especie a la dieta, en contra de lo comúnmente aceptado, el consumo de bovinos habría sido el más importante, desplazando al ciervo a un segundo plano en el Asturiense Inicial y Neolítico; (2) se confirma la tendencia progresiva hacia una mayor diversidad de la dieta animal, eviden- ciada en la incorporación significativa del jabalí y, más residualmente, del corzo, identificándose también un consumo ocasional de pequeños carnívoros; (3) a partir del estudio de los perfiles esqueléticos de ciervo, se verifica el carácter residencial de la ocupación de la base del conchero, en el que se habrían consumido las carcasas cazadas (la escasez de restos de otros taxones ha impedido su estudio); y (4) el perfil de edades de muerte de los ungulados, sobre todo en momentos iniciales del Asturiense podría catalogarse como "catastrófico" al implicar un consumo mayoritario de individuos infantiles y juveniles.

En este trabajo se ha considerado representativo abordar la importancia de los ungulados en la dieta desde dos puntos de vista metodológicos diferentes: el método clásico de utilización del NR y otro alternativo de estimación de biomasa en función de la edad y el Número Mínimo de Individuos representados. Los resultados han mostrado que, atendiendo exclusivamente a la representación del NR, el ciervo es el animal más importante en todos los períodos, lo que estaría en línea con lo hasta ahora establecido. Sin embargo, esta situación cambia a partir de la cantidad de carne que pudo haberse aprovechado de cada uno de los animales identificados. Los resultados son diametralmente opuestos para el Asturiense Inicial y Neolítico, donde los bovinos relegan al ciervo como el taxón más representativo, lo que introduce elementos de incertidumbre en la metodología clásica hasta ahora desarrollada. Por ejemplo, en el Asturiense Inicial, la elevada cantidad de restos de ciervo no tendría por qué haberse correspondido con el mayor aporte calórico de la dieta, pues el $67 \%$ de los individuos son infantiles, aportando una reducida cantidad alimenticia en comparación con los bovinos, que corresponden a juveniles y adultos. No obstante, tampoco la estimación del NMI está exenta de errores $\mathrm{y}$, en general, tiende a sobrerrepresentar las especies con menor número de restos, posiblemente las menos consumidas. Además, no se puede establecer a priori si los animales han sido aportados enteros o no al yacimiento, lo que introduce un nuevo elemento de potencial error en la estimación de la biomasa. En este contexto, la mejor opción consistiría en la adopción de ambas metodologías como posible acotación de la dieta real.

En cualquier caso, y como consecuencia de los resultados obtenidos, se puede definitivamen- 
te refutar que los concheros fueron originados como basureros derivados de la explotación episódica del litoral por parte de grupos del interior (Straus 1979), teoría que, tras los trabajos de Fano (1998) sobre la intencionalidad de su ubicación en lugares soleados y resguardados y el descubrimiento de yacimientos de interior (p.e. Los Canes), había dejado ya relegada. Se trataría, por tanto, de los campamentos de habitación de los grupos humanos que habitaron la costa, ya que, si bien podrían haber existido asentamientos al aire libre, este extremo no ha podido ser todavía demostrado. A su vez, la progresiva transgresión marina, al anegar la plataforma litoral, podría haber ocultado otros yacimientos costeros (Fano 2004), aunque hay que señalar el hecho de que, hacia los $9 \mathrm{ka} \mathrm{BP}$, el nivel del mar se encontraría sólo unos $18 \mathrm{~m}$ por debajo del actual y hacia los 6 ka BP la línea de costa habría ya alcanzando su configuración moderna (Bailey y Craighead 2004), por lo que potencialmente este fenómeno debería haber afectado más intensamente a la visibilidad de yacimientos del final del Pleistoceno.

En este sentido, los testimonios arqueológicos disponibles en la actualidad podrían considerarse el reflejo directo de la estrategia de subsistencia de los últimos cazadores-recolectores $\mathrm{y}$, de esta forma, se confirmaría, por un lado, una marcada oscilación en el aporte de mamíferos terrestres a la dieta. Este hecho se ha podido comprobar al calcular el índice de la representación de los ungulados en la dieta, como el cociente entre NR total por períodos de cada yacimiento con estudios de fauna, dividido por el número total de niveles arqueológicos. Así, se pasaría de un promedio por nivel arqueológico de 912,3 en el conjunto de yacimientos magdalenienses en toda la Región Cantábrica, a aproximadamente 1360,3 en el Aziliense a un marcado descenso a 201,3 en el Mesolítico. Por otro lado, un aprovechamiento integral del medio, con un incremento significativo del nicho ecológico, incorporando masivamente recursos hasta ahora complementarios. Así, en la bibliografía son comunes las referencias al consumo de aves en niveles mesolíticos (Arias 1992; Clark 1976; Elorza 1990), a la incorporación de recursos vegetales, posiblemente de avellanas y bellotas, apoyado por el registro antracológico de Mazaculos (Uzquiano 1995; Zapata 2000) y por los análisis dentarios de los restos humanos de Aizpea (Rúa et al. 2001), al aporte de caracoles terrestres (p.e. en Cubio Redondo, Aparicio 2001), La Fragua (6) y a la progresiva importancia de la pesca, tanto fluvial como marina (Menéndez de la Hoz et al. 1986; Roselló 1990). Pero sin duda, es el marisqueo la actividad diferenciadora de este período. Si bien durante el Tardiglacial ya se documentan concheros, es en el Mesolítico cuando los restos de moluscos, crustáceos y equinodermos sufren un notable incremento. Los concheros asturienses incluyen típicamente lapas (fundamentalmente Patella vulgata y Patella intermedia), bígaros (Osilinus lineatus), erizos y mejillones. En el Cantábrico oriental se documentan también almejas y ostras en abundancia (Fano 2004).

No sólo se manifiesta un aumento del aprovechamiento de recursos marinos durante el Mesolítico, sino que las evidencias malacológicas avalan además una ampliación de las zonas explotadas. Se ha documentado en La Riera (Ortea 1986), Poza L'Egua (Arias et al. 2007b) y La Fragua (Gutiérrez Zugasti 2006) un incremento de Patella intermedia (propia de zonas más expuestas, frente a Patella vulgata, asociada a estuarios y zonas resguardadas) y una reducción progresiva del tamaño de los individuos, interpretada como resultado de una sobreexplotación debida a la presión demográfica (Clark y Straus 1986) o por la variación de la temperatura del mar (Bailey y Craighead 2004). En definitiva, la dieta humana sufre un cambio drástico respecto al final del Paleolítico, fundamentalmente caracterizado por un intercambio del papel otorgado a los recursos terrestres y marinos (Arias y Fano 2005).

El significado de los testimonios disponibles puede ser interpretado como un intento de los grupos humanos de reducir riesgos y establecer una estrategia de explotación del medio más sostenible (Arias 1991) o como la respuesta lógica a la progresiva reforestación y subida del nivel del mar (Bailey 1983). Sin embargo, un análisis más detallado del fenómeno, revelaría la adopción de conductas económicas menos productivas (no sólo el aporte calórico de los recursos marinos o vegetales es inferior a la de los mamíferos te-

(6) Gutiérrez Zugasti, I. 2005. La explotación de moluscos en la cuenca baja del río Asón (Cantabria, España) a inicios del Holoceno (10,000-5,000 BP) y su importancia en las comunidades humanas del Aziliense y del Mesolítico. Trabajo de Investigación. Universidad de Cantabria. Santander. 
rrestres (USDA 2008) sino que su adquisición supone, según algunos autores, un gasto energético superior, lo que conduce a una menor ratio de energía neta (Bettinger et al. 1997; Bird y Bliege-Bird 1997; Jones y Madsen 1989; Metcalfe y Barlow 1992), algo que, a la luz de los postulados de la Teoría del Forrajeo Óptimo (MacArthur y Pianka 1966; Emlen 1966), sería perjudicial para la propia supervivencia (Kaplan y Hill 1992). Desde esa perspectiva, esta estrategia únicamente sería adoptada cuando se agotaran las opciones para una dieta especializada en mamíferos terrestres. Es decir, sólo cuando la abundancia de éstos se redujese de forma que sus costes de adquisición aumentasen hasta el punto de que su productividad descendiera por debajo de un determinado umbral, se incluirían en la dieta otros elementos de menor contribución energética neta (Davis 2005; Gassiot 2000 (7); Marín Arroyo 2008c).

El argumento anterior ya fue esgrimido por Freeman (1981), Clark (1987), Clark y Straus (1986), Clark y Yi (1983) y Davis et al. (1988) como confirmación del papel del crecimiento demográfico en el paso de una economía especializada en ciervo y cabra durante el Solutrense y sobre todo Magdaleniense, a otra más diversificada en el Aziliense y Mesolítico, y retomado por Marín Arroyo (2008c) como contrapunto a las tesis ambientalistas mayoritarias. Sin embargo, lo que distingue al Mesolítico Cantábrico, a partir del registro arqueológico disponible, es la dramática reducción de restos de fauna terrestre, resultando en unos testimonios económicos muy pobres como para poder esgrimir la existencia de una población elevada en la región (Straus 2004). Como uno de nosotros (8) demuestra a través de una modelización matemática de la interacción de las poblaciones de ungulados y los grupos humanos, existe una distancia reducida entre la posibilidad de una densidad demográfica elevada sustentada sobre una dieta variada de mamíferos terrestres y la ocurrencia de una masiva extinción de las especies depredadas, en un escenario de gran presión cinegética y cambios ambientales bruscos,

(7) Gassiot, E. 2000. Anàlisi arqueológica del canvi cap a l'explotación del litoral. Tesis doctoral inédita. Universidad Autónoma de Barcelona. Barcelona.

(8) Marín Arroyo, A.B. 2007. La fauna de mamíferos en el Cantábrico Oriental durante el Magdaleniense y Aziliense: nuevos enfoques y líneas de investigación arqueozoológica. Tesis doctoral inédita. Universidad de Cantabria. Santander. que, dada la imposibilidad del resto de recursos del medio para abastecer a la población alcanzada, derivaría en una crisis alimenticia y demográfica grave.

En ese contexto, podría bastar la sucesión de varios inviernos duros, con nevadas intensas, para provocar una marcada debilitación y posible extinción de los rebaños de ciervos como sucedió en 1979 en los montes de Erregerena, Navarra (Mairezkurrena 1983) y, en consecuencia, el colapso en cascada del resto de poblaciones de ungulados (Estévez 2005). La existencia de un perfil de edades catastrófico en los niveles de Asturiense inicial de Mazaculos podría apoyar la idea de una reducción repentina de las poblaciones animales según los argumentos de este último autor.

Siguiendo esa misma línea de investigación, el hiato en las dataciones de radiocarbono identificado en el Cantábrico entre 10,12 y 9,6 ka BP (Cueto et al. 2005/2006) coincide con una fase de enfriamiento brusco provocada por la rotura repentina del dique del lago Agassiz que habría derivado en la brusca parada de la corriente noratlántica (Clark et al. 2001; Colman 2002) y la del dique del Báltico, motivando en conjunto una bajada de la temperaturas de $4^{\circ} \mathrm{C}$ en 100 años durante la oscilación del Preboreal, recuperada posteriormente tras un período de ascenso continuado en 50 años (Björck et al. 1998). Este posible colapso demográfico podría explicar la brusca ruptura económica y tecnológica apreciada. Así, los grupos azilienses se habrían enfrentado en sus momentos finales a una crisis alimenticia severa que probablemente habría potenciado la emigración. La población remanente, de reducida cuantía, podría haber adoptado tácticas económicas diferentes, a través de la intensificación del uso del medio marino tras el soporte perdido en los mamíferos terrestres.

En lo que respecta a los inicios de la domesticación, el yacimiento de Mazaculos aporta también datos interesantes. Reconocer su existencia en conjuntos de transición no es una labor fácil para el arqueozoólogo. En el caso que nos corresponde, se ha podido evidenciar la presencia de animales domésticos (en el ganado ovino y bovino), si bien, su importancia económica parece todavía débil. En cualquier caso, se trata de las primeras pruebas económicas documentadas en el Cantábrico occidental, con una datación de $5050 \pm 120$ BP. 


\section{AGRADECIMIENTOS}

En primer lugar queremos agradecer a Jesús Altuna la labor que realizó en el análisis preliminar de los restos del nivel 3. Pedro Castaños nos ha brindado sus acertadas observaciones sobre el inicio de la domesticación en la Cornisa Cantábrica. Arturo Morales nos abrió su laboratorio permitiéndonos comparar algunos de los materiales aquí estudiados. Jordi Estévez nos aportó comentarios críticos que permitieron mejorar este trabajo e Igor Gutiérrez aportó indicaciones oportunas en la redacción final. Debemos agradecer igualmente la colaboración de la Sociedad de Ciencias Aranzadi por darnos acceso a su colección osteológica. Por último, gracias a David Ocio por su apoyo incondicional en todo momento sin esperar nada a cambio.

\section{BIBLIOGRAFÍA}

Alados, C.L. y Escós, J. 2003: “Cabra montés - Capra pyrenaica”. En L.M. Carrascal y A. Salvador (eds.): Enciclopedia Virtual de los Vertebrados Españoles. Museo Nacional de Ciencias Naturales. Madrid. http://www.vertebradosibericos.org

Altuna, J. 1972: "Fauna de mamíferos de los yacimientos prehistóricos de Guipúzcoa". Munibe 24: $1-464$.

Altuna, J. 1979: "La faune des Ongulés du Tardiglaciaire en Pays Basque et dans le reste de la région Cantabrique". Colloques Internationaux CNRS 271. La Fin des Temps glaciaire en Europe (París, 1977): 85-96. Burdeos.

Altuna, J. 1980: "Historia de la domesticación animal en el País Vasco desde sus orígenes a la romanización". Munibe 32: 1- 163.

Altuna, J. 1986: "The mammalian faunas from the Prehistoric Site of La Riera". En L.G. Straus y G. Clark (eds.): La Riera Cave. Stone Age Hunter-Gatherer adaptations in Northern Spain. Anthropological Research Paper 36. Arizona: 237-274; 421-479; 481-497.

Altuna, J. 1995: "Fauna de mamíferos y cambios ambientales durante el Tardiglacial cantábrico". En A. Moure y C. González Sainz (eds.): El Final del Paleolítico cantábrico. Transformaciones ambientales y culturales durante el Tardiglaciar y comienzos del Holoceno en la Región Cantábrica. Servicio de Publicaciones de la Universidad de Cantabria. Santander: 77-117.

Altuna, J. y Mariezkurrena, K. 1984: "Bases de subsistencia de origen animal en el yacimiento de Ekain". En J. Altuna y J.M. Merino (dir.): El yacimiento prehistórico de la Cueva de Ekain (Deba, Guipúzcoa). Sociedad de Estudios Vascos, serie B 1. San Sebastián: 211-280.

Altuna, J.; Cuenca Bescós, G.; Elorza, M.; García Pimienta, J.C.; Lobo, J.; Mariezkurrena, K.; Pérez Ripoll, M.; Sánchiz, B.; González Morales, M. y Straus, L.G. 2004: "Post-pleistocene faunas from the archeological site of El Mirón cave (Ramales de la Victoria, Cantabria, Spain): a preliminary summary". Zona arqueológica 4 (2): 40-49.

Aparicio, M.'T. 2001: "Malacofauna terrestre del yacimiento de Cubio Redondo (Matienzo, Cantabria)". Munibe 53: 61-66.

Arias, P. 1991: De cazadores a campesinos. La transición al neolítico en la región cantábrica. Servicio de Publicaciones de la Universidad de Cantabria-Asamblea Regional de Cantabria. Santander.

Arias, P. 1992: "Estrategias económicas de las poblaciones del Epipaleolítico avanzado y el Neolítico en la región cantábrica". En A. Moure (ed.): Elefantes, ciervos y ovicaprinos: economía y aprovechamiento del medio en la Prehistoria de España y Portugal. Servicio de Publicaciones de la Universidad de Cantabria. Santander: 163-184.

Arias, P. y Altuna, J. 1999: "Nuevas dataciones absolutas para el Neolítico de la cueva de Arenaza (Bizkaia)". Munibe 51: 161-171.

Arias, P. y Fano, M. 2005: "Le rôle des ressources marines dans le Mésolitique de la région Cantabrique (Espagne): l'apport des isotopes stables". En G. Marchand y A. Tresset (eds.): Unité y diversité des processus de néolithisation sur la façade atlantique de l'Europe. Mémoires de la Société Préhistorique Française 36. Paris: 173-188.

Arias, P. y Pérez, C. 1995: "Excavaciones arqueológicas en Arangas, Cabrales (1991-94): Las Cuevas de Los Canes, el Tiu Llines y Arangas". Excavaciones arqueológicas en Asturias 1991-94 (3): 79-92.

Arias, P.; Fano, M.; Armendariz, A.; Álvarez, E.; Cueto, M.; Garralda, M.D.; Mensua, C. y Teira, L. 2007a: "Programa de sondeos en concheros holocenos del Oriente de Asturias". Excavaciones arqueológicas en Asturias 1999-2002: 107-116.

Arias, P.; Fernández-Tresguerres, J.; Álvarez, E.; Armendáriz, A.; Cueto, M.; Fano, M.; Fernández, R.; Garralda, M.D.; Mensua, C. y Teira, L. 2007b: "Excavación arqueológica de urgencia en la cueva de Poza L'Egua Lledías, Llanes”. Excavaciones arqueológicas en Asturias 1999-2002: 227-239.

Arnold, E.R. y Greenfield, H.J. 2006: The Origins of Transhumant Pastoralism in Temperate Southeastern Europe: A Zooarchaeological Perspective from the Central Balkans. British Archaeological Reports, International Series 1538. Oxford.

Aura, J.; Villaverde, V.; González Morales, M.R.; González Sainz, C.; Zilhão, J. y Straus, L.G. 1998: "The Pleistocene-Holocene transition in the iberian 
peninsula: continuity and change in human adaptations". Quaternary International 49-50: 87-103.

Azorit, C.; Analla, M.; Carrasco, R.; Calvo, J.A. y Muñoz-Cobo, J. 2002: "Teeth eruption pattern in red deer (Cervus elaphus hispanicus) in southern Spain". Anales de Biología 24: 107-114.

Bailey, G.N. 1978: "Shells middens as indicators of postglacial economies: a territorial perspective". En P. Mellars (ed.): The early postglacial settlement of northern Europe. Duckworth. London: 37-63.

Bailey, G.N. 1983: "Economic change in late pleistocen Cantabria". En G.N. Bailey (ed.): Hunter-gatherer economy in prehistory. Cambridge University Press. Cambridge: 149-165.

Bailey, G. y Craighead, A. 2004: "Coastal Paleoeconomies and Paleoenviromental Trends: Asturian and Australian middens compared". En M.R. González Morales y G.A. Clark (eds.): The Mesolithic of the Atlantic Façade: Proceedings of the Santander Symposium. Anthropological Research Papers 55. Arizona: 181-204.

Bartram, L.E. y Marean, C.W. 1999: "Explaining the 'Klasies pattern': Kua ethnoarchaeology the Die Kelders Middle Stone Age archaeofauna, long bone fragmentation and carnivore ravaging". Journal of Archaeological Science 26: 9-29.

Berganza, E. 1990: "El Epipaleolítico en el País Vasco". Munibe 42: 81-89.

Bettinger, R.L.; Malhi, R.S. y McCarthy, H. 1997: "Central place models of acorn and mussel processing". Journal of Archaeological Science 24: 887-899.

Binford, L.R. 1978: Nunamiut Ethnoarchaeology. Academic Press. New York.

Binford, L.R. 1981: Bones: Ancient Men and Modern Myths. Academic Press. New York.

Bird, D.W. y Bliege Bird, R. 1997: “Contemporary shellfish gathering strategies among the Merian of the Torres Straits Islands, Australia: testing predictions of a central place foraging model'. Journal of Archaeological Science 24: 39-63.

Björck, S.; Walker, M.J.C.; Cwynar, L.C.; Johnsen, S.; Knudsen, K.L.; Lowe, J.J.; Wohlfarth, B. e Intimate members 1998: "An event stratigraphy for the Last Termination in the North Atlantic region based on the Greenland ice-core record: a proposal by the Intimate group". Journal of Quaternary Science 13: 283-292.

Broughton, J.M. 2002: "Prey spatial structure and behaviour affect archaeological tests of optimal foraging models: examples from the Emeryville Shellmound Vertebrae Fauna". World Archaeology 34 (1): 60-83.

Bull, G. y Payne, S. 1982: "Tooth eruption and epiphysial fusion in pigs and wild boar". En B. Wilson, C. Grigson y S. Payne (eds.): Ageing and Sexing
Animal Bones from Archaeological Sites. British Archaeological Reports, British Series 109. Oxford: $55-71$.

Carranza, J. 2004: “Ciervo - Cervus elaphus". En L.M. Carrascal y A. Salvador (eds.): Enciclopedia Virtual de los Vertebrados Españoles. Museo Nacional de Ciencias Naturales. Madrid. http://www.vertebradosibericos.org

Castaños, P. 1980: "La macrofauna de la cueva de La Paloma (Pleistoceno Terminal de Asturias)". En M. Hoyos, I. Martínez, T. Chapa, P. Castaños y F.B. Sánchiz, La Cueva de La Paloma (Soto de las Regueras, Asturias). Excavaciones Arqueológicas en España 116: 65-100.

Castaños, P. 1982: "Estudio de los macromamíferos del yacimiento prehistórico de Cueto de la Mina (Asturias)". Boletín del Instituto de Estudios Asturianos: 43-86, 105-106.

Castaños, P. 1998/2000: "Estudio de los restos faunísticos del yacimiento de Kobeaga II". Illunzar 4: 173-175.

Castaños, P. 2001: "Estudio arqueozoológico de la fauna del yacimiento de Cubio Redondo (Matienzo, Cantabria)". Munibe 53: 71-74.

Castaños, P. 2004: “Estudio arqueozoológico de los macromamíferos del Neolítico de la Cueva de Chaves (Huesca)". Saldvie 4: 125-171.

Clark, G.A. 1976: El Asturiense Cantábrico. Bibliotheca Praehistorica Hispana XIII. CSIC. Madrid.

Clark, G.A. 1987: "From the Mousterian to the Metal Ages: long-term change in the human diet of Cantabrian Spain". En O. Soffer (ed.): The Pleistocene Old World: a regional perspectives. Plenum. New York: 293-316.

Clark, G.A. 1995: “Complementariedad funcional en el Mesolítico del Norte de España”. En V. Villaverde (ed.): Los últimos cazadores. Transformaciones culturales y económicas durante el Tardiglariar y el inicio del Holoceno en el ámbito mediterráneo. Instituto de Cultura Juan Gil-Albert y Diputación de Alicante. Alicante: 63-78.

Clark, G.A. 2004: "The Iberian mesolithic in the European context". En M.R. González Morales y G.A. Clark (eds.): The Mesolithic of the Atlantic Façade: Proceedings of the Santander Symposium. Anthropological Research Papers 55. Arizona: 205-224.

Clark, G.A. y Clark, V.J. 1975: "La cueva del Balmori Asturias, España: nuevas aportaciones". Trabajos de Prehistoria 32: 25-77.

Clark, G.A. y Lerner, S. 1980: "Prehistoric resource utilisation in early Holocene Cantabrian Spain". Anthropology UCLA 10: 53-96.

Clark, G.A. y Straus, L.G. 1983: "Late Pleistocene hunter-gatherer adaptations in Cantabrian Spain". En G. Bailey (ed.): The Pleistocene Old World: a 
European perspective. Cambridge University Press. Cambridge: 131-148.

Clark, G.A. y Straus, L.G. 1986: "Synthesis and conclusions - Part I: Upper Palaeolithic and Mesolithic hunter-gatherer subsistence in northern Spain". En L.G. Straus y G.A. Clark (eds.): La Riera Cave: Stone Age Hunter-Gatherer Adaptations in northern Spain. Arizona State University Anthropological Research Papers 36. Tempe: 351-366.

Clark, G.A. y Yi, S. 1983: "Niche-width variation in Cantabrian archaeofaunas". En J. Clutton Brock y C. Grigson (eds.). A diachronic study. Animals and Archaeology I: Hunters and their prey. British Archaeological Reports. International Series 163, Oxford: 183-208.

Clark, P.; Marshall, S.J.; Shawn, M.J.; Clarke, G.K.; Hostetler, S.W.; Licciardi, J. y Teller, J. 2001: "Freshwater forcing of abrupt climate change during the Last Glaciation". Science 293 (5528): 283-287.

Cleghorn, N. y Marean, C.W. 2004: "Distinguishing selective transport and in situ attrition: a critical review of analytical approaches". Journal of Taphonomy 2: 43-67.

Colman, S.M. 2002: "A fresh look at Glacial floods". Science 296 (5571): 1251-1252.

Cueto, M.; Marín, A.B. y Estévez, J. 2005/2006: "Apuntes para un cambio de ritmo en la explicación del cambio al Postglaciar". Munibe (Antropologia-Arkeologia). Homenaje al Prof. Jesús Altuna 57 (1): 399-410.

Davis, S. 2005: "Why domesticate food animals? Some zoo-archaeological evidence from the Levant". Journal of Archaeological Science 32: 1408-1416.

Davis, S.; Rabinovick, R. y Gren-Inbar, N. 1988: "Quaternary Extinctions and population increase in western Asia: the animal remains from Big'at Quneitra". Paléorient 14 (1): 95-105.

Elorza, M. 1990: "Restos de aves en los yacimientos prehistóricos vascos. Estudios realizados". Munibe 42: 263-267.

Emlen, J.M. 1966: "The role of time and energy in food preferences". American Naturalist 100: 611-617.

Estévez, J. 2005: Catástrofes en Prehistoria. Editorial Bellaterra. Barcelona.

Faith, J.T. y Gordon, A.D. 2007: "Skeletal element abundance in archaeofaunal assemblages: economic utility, sample size, and assessment of carcass transport strategies". Journal of Archaeological Science 34: 872-882.

Fano, M. 1996: "El mesolítico en Asturias: delimitación cronológica y espacial". Complutum 7: 51-62.

Fano, M. 1998: El hábitat mesolitico en el Cantábrico occidental. Transformaciones ambientales y medio físico durante el Holoceno antiguo. British
Archaeological Reports, International Series 732. Oxford.

Fano, M. 2000: "Después del Asturiense: ocho décadas de incertidumbre acerca del inicio del Neolítico en el Cantábrico occidental". Veleia 17: 9-30.

Fano, M. 2004: "Un nuevo tiempo: el mesolítico en la Región Cantábrica". Kobie, Anejo 8: 337-402.

Fernández-Llario, P. 2006: “Jabalí - Sus scrofa”. En L.M. Carrascal y A. Salvador (eds.): Enciclopedia Virtual de los Vertebrados Españoles. Museo Nacional de Ciencias Naturales. Madrid. http://www.vertebradosibericos.org.

Fernández-Tresguerres, J.A. 1983: "Visión general del epipaleolítico cantábrico". En Homenaje al Prof. Martín Almagro Basch I. Ministerio de Cultura. Madrid: 131-137.

Freeman, L.G. 1973: "The significance of mammalian faunas from Palaeolithic occupations in Cantabrian Spain". American Antiquity 38: 3-44.

Freeman, L.G. 1981: "The Fat of the Land: notes on Palaeolithic Diet in Iberia". En R.S.O. Harding y G. Teleki (eds.): Omnivorous Primates. Gathering and hunting in human evolution. Columbia University Press. New York: 104-165.

González Morales, M.R. 1978: “Excavaciones en el conchero asturiense de Mazaculos II (La Franca, Ribadedeva, Asturias)". Boletín del Instituto de Estudios Asturianos 93-94: 363-383.

González Morales, M.R. 1982: El Asturiense y otras culturas locales. La explotación de las áreas litorales de la región cantábrica en los tiempos epipaleolíticos. Monografías del Centro de Investigación y Museo de Altamira 7. Ministerio de Cultura. Madrid.

González Morales, M.R. 1992: “Mesolíticos y megalíticos: la evidencia arqueológica de los cambios en las formas productivas en el paso al megalitismo en la costa cantábrica”. En A. Moure (ed.): Elefantes, ciervos y ovicaprinos: economía y aprovechamiento del medio en la Prehistoria de España y Portugal. Servicio de Publicaciones de la Universidad de Cantabria. Santander: 185-202.

González Morales, M.R. 1995a: "La transición al Holoceno en la Región Cantábrica: el contraste con el modelo del mediterráneo español". En V. Villaverde (ed.): Los últimos cazadores. Transformaciones culturales y económicas durante el Tardiglariar y el inicio del Holoceno en el ámbito mediterráneo. Instituto de Cultura Juan Gil-Albert y Diputación de Alicante. Alicante: 63-78.

González Morales, M.R. 1995b: "Memoria de los trabajos de limpieza y toma de muestras en los yacimientos de las Cuevas de Mazaculos y El Espinoso La Franca, Ribadedeva y La Llana (Andrín, Llanes) en 1993". Excavaciones Arqueológicas en Asturias, 1991-94 (3): 65-78. 
González Morales, M.R. 1999: “Costa e interior: algunas observaciones sobre el uso de las cuevas en el Mesolítico". En De Oriente a Occidente, Homenaje al Dr. Emilio Olavarri. Universidad Pontificia de Salamanca. Salamanca: 237-246.

González Morales, M.R. 2000: "La Prehistoria de las Marismas: Excavaciones en la Cueva de la Fragua (Santoña). Campañas de 1990, 1991, 1993, 1994 y 1996". En R. Ontañón (ed.): Actuaciones Arqueológicas en Cantabria 1984-1999. Consejería de Cultura y Deporte. Santander: 93-96.

González Morales, M.R. y Díaz Casado, Y. 1991/92: "Excavaciones en los abrigos de la Peña del Perro Santoña, Cantabria. Estratigrafía, cronología y comentario preliminar de sus industrias". Veleia 8-9: 43-64.

González Morales, M.R. y Márquez Uría, M. 1978: "The Asturias shell midden of Cueva de Mazaculos II". Current Anthropology 19 (3): 614-615.

González Morales, M.R.; Márquez Uría, M.C.; Daz, T.E.; Ortea Rato, J.A. y Volman, K. 1980: "Informe preliminar de las excavaciones en el conchero asturiense de la cueva de Mazaculos II La Franca, Asturias: Campañas de 1976-78". Noticiario Arqueológico Hispánico 9: 35-62.

González Sainz, C. 1989: El Magdaleniense Superior Final de la región Cantábrica. Ediciones Tantín. Santander.

Gutiérrez Zugasti, I. 2006: “Análisis arqueomalacológico de la Cueva de La Fragua (Santoña, Cantabria, España)". En N. Ferreira Bicho (ed.): Animais na Pre-História e Arqueología da Península Ibérica (Faro, 2004). Actas do IV Congresso de Arqueología Penínsular: 197-210. Faro. Portugal.

Iriarte, M.J.; Mujika, J. y Tarriño, A. 2005: “Herriko Barra (Zarautz-Guipuzkoa): Caractérisation industrielle et économique des premiers groupes de producteurs sur le littoral basque”. En G. Marchand y A. Tresset (eds.): Unité et diversité des processus de néolithisation sur la façade atlantique de l'Europe. Mémoires de la Société Préhistorique Française 36. Paris: 127-136.

Jones, K.T. y Madsen, D.B. 1989: "Calculating the cost of recourse transportation: a Great Basin example". Current Anthropology 30 (4): 529-534.

Kaplan, H. y Hill, K. 1992: "The evolutionary ecology of food acquisition". En E.A. Smith y B. Winterhalder (eds.): Evolutionary ecology and Human behaviour. Aldine de Gruyter. New York: 167-201.

Klein, R.G. y Cruz-Uribe, K. 1984: The Analysis of Animal Bones from Archaeological Sites. The University of Chicago Press. Chicago.

Lam, Y.M.; Chen, X. y Pearson, O.M. 1999: "Intertaxonomic variability in patterns of bone density and the differential representation of bovid, cervid, and equid elements in the archaeological record". American Antiquity 64 (2): 343-362.
Lyman, R.L. 1994: Vertebrate Taphonomy. Cambridge University Press. Cambridge.

Macarthur, R. y Pianka, E.R. 1966: "On optimal use of a patchy environment". American Naturalist 100: 603-609.

Marean, C.W. y Cleghorn, N. 2003: "Large mammal skeletal element transport: applying foraging theory in a complex taphonomic system". Journal of Taphonomy 1: 15-42.

Marean, C.W.; Abe, Y.; Nilssen, P. y Stone, E. 2001: "Estimating the minimum number of skeletal elements MNE in zooarchaeology: a review and a new image-analysis GIS Approach". American Antiquity 66: 333-348.

Marean, C.W.; Domínguez-Rodrigo, M. y Pickering, T. R. 2004: "Skeletal element equifinality in zoorchaeology begins with method: the evolution and status of the "shaft critique". Journal of Taphonomy 2: 69-98.

Mariezkurrena, K. 1983: “Contribución al conocimiento del desarrollo de la dentición y el esqueleto postcraneal de Cervus elaphus". Munibe 35: 149-202.

Mariezkurrena, K. y Altuna, J. 1995: "Fauna de mamíferos del yacimiento costero de Herriko Barra (Zarautz, País Vasco)". Munibe 47: 23-32.

Marín Arroyo, A.B. 2004: Análisis arqueozoológico, tafonómico y de distribución espacial de la fauna de mamíferos de la Cueva de La Fragua (Santoña, Cantabria). Ediciones TGD. Santander.

Marín Arroyo, A.B. 2008a: "Patrones de movilidad y control del territorio en el Cantábrico Oriental durante el Tardiglacial". Trabajos de Prehistoria 65 (1): 29-45.

Marín Arroyo, A.B. 2008b: "El yacimiento paleolítico de la Cueva del Mirón: Resultados de la aplicación de nuevas metodologías arqueozoológicas". En C. Díez (ed.): Zooarqueología hoy. Encuentros hispano-argentinos. Servicio de Publicaciones de la Universidad de Burgos. Burgos: 69-87.

Marín Arroyo, A.B. 2008c: "Demografía y cambio ambiental. Hipótesis para el cambio económico en el cantábrico oriental durante el Tardiglacial". Complutum 19 (1): 47-66.

Marín Arroyo, A.B. 2009a: "The human use of the montane zone of Cantabrian Spain during the Late Glacial: faunal evidence from El Mirón Cave". Journal of Anthropological Research 65 (1): 69-102.

Marín Arroyo, A.B. 2009b: "A practical comparative study of the available analytic techniques for skeletal part profile interpretation at El Mirón Cave (Cantabria, Spain)". Archaeofauna 18.

Marín Arroyo, A.B. 2009c: "Economic adaptations during the Late Glacial in northern Spain. A simulation approach". Before Farming, issue 3. 
Mateos-Quesada, P. 2005: “Corzo - Capreolus capreolus". En L.M. Carrascal y A. Salvador (eds.): Enciclopedia Virtual de los Vertebrados Españoles. Museo Nacional de Ciencias Naturales. Madrid. http://www.vertebradosibericos.org

Menéndez de la Hoz, M.; Straus, L.G. y Clark, G.A. 1986: "The icthyology of La Riera cave". En L.G. Straus y G.A. Clark (eds.): La Riera Cave. Stone Age hunter-gatherers in Northern Spain. Anthropological Research Papers 36. Arizona State Univesity. Tempe: $285-288$.

Metcalfe, D. y Barlow, K.R. 1992: "A model for exploring the optimal trade-off between field processing and transport". American Anthropologist 94 (2): 340-356.

Monahan, C. 1998: "The Hadza carcass transport debate revised and its archaeological implications". Journal of Archaeological Science 25: 404-424.

Munro, N.D. 2004: "Zooarchaeological measures of hunting pressure and occupation intensity in the Natufian”. Current Anthropology 45 (Supplement): 5-33.

Muñoz, M. y Berganza, E. 1997: El yacimiento de la cueva de Urratxa III (Orozko, Bizkaia). Universidad de Deusto. Bilbao.

O’Connell, J.F.; Hawkes, K. y Blurton Jones, N. 1988: "Hadza hunting, butchering, and bone transport and their archaeological implications". Journal of Anthropological Research 44: 113-161.

Ontañón, R. 2005: “La secuencia de la Cueva de Los Gitanos (Castro Urdiales, Cantabria) y el Neolítico Cantábrico". En P. Arias; R. Ontañón y C. García-Monco (eds.): III Congreso del Neolítico en la Península Ibérica (Santander, 2003): 1035-1043. Santander.

Ontañón, R.; Moure, J.A.; Arias. P. y González Sainz, C. 2000: "La Zona Arqueológica de la Garma (Cantabria): investigación, conservación y uso social". Trabajos de Prehistoria 57 (2): 41-56.

Ortea, J. 1986: "The malacology of La Riera cave". En L.G. Straus y G.A. Clark (eds.): La Riera Cave. Stone Age hunter-gatherers in Northern Spain. Anthropological Research Papers 36. Arizona State Univesity. Tempe: 289-298.

Pales, L. y Lambert, C. 1972: Atlas ostéologiques des mammifères. CNRS. París.

Peña-Chocarro, L.; Zapata, L.; González Morales, M.R.; García Gazolaz, J.; Sesma Sesma, J. y Straus, L.G. 2005: "The spread of agriculture in northern Iberia: new archaeobotanical data from El Mirón Cave (Cantabria) and the open-air site of Los Cascajos (Navarra)". Vegetation History and Archaeobotany 14: 268-278.

Pérez-Barbería, F.J. y García-González, R. 2004: “Rebeco - Rupicapra pyrenaica". En L.M. Carrascal y A. Salvador (eds.): Enciclopedia Virtual de los Verte- brados Españoles. Museo Nacional de Ciencias Naturales. Madrid. http://www.vertebradosibericos.org.

Pérez Ripoll, M. 1992: Marcas de carnicería, fracturas intencionadas y mordeduras de carnívoros en huesos prehistóricos del Mediterráneo español. Instituto de Cultura Juan Gil-Albert. Alicante.

Pérez Suárez, C. 1995: "Carta arqueológica de los concejos de Llanes y Ribadedeva (1992)". Excavaciones arqueológicas en Asturias 1991-94 (3): 243-245.

Pickering, T.; Marean, C.W. y Domínguez-Rodrigo, M. 2003: "Importance of limb bone shaft fragments in zooarchaeology: a response to 'On in situ attrition and vertebrate body part profiles' 2002” by M.C. Stiner. Journal of Archaeological Science 30: 1469-1482.

Purdue, J.R. 1983: "Epiphyseal closure in white-tailed deer". Journal of Wildlife Management 47 (4): 1207-1213.

Ruiz Cobo, J. y Smith, P. 2001: The archaeology of the Matienzo Depresión, North Spain. British Archaeological Reports, International Series No. 975. Oxford.

Rogers, A.R. 2000a: “On equifinality in faunal analysis". American Antiquity 65: 709-723.

Rogers, A.R. 2000b: "Analysis of bone counts of maximum likelihood". Journal of Archaeological Science 27: 111-125.

Roselló Izquierdo, E. 1990: Arqueoictiofauna ibéricas. Aproximación metodológica y biocultural. Universidad Autónoma de Madrid. Madrid.

Rúa, C. de la; Baranybar, J.; Iriondo, M. y Izaguirre, N. 2001: "Estudio antropológico del esqueleto mesolítico del yacimiento de Aizpea". En I. Barandiarán y A. Cava (dir.): Cazadores-recolectores en el Pirineo Navarro. El sitio de Aizpea entre 8.000 y 6.000 años antes de ahora. Anejos de Veleia 10. Universidad del País Vasco. Vitoria: 363-429.

Schmid, E. 1972: Atlas of Animal Bones. Elsevier Publishing Company. Amsterdam.

Shannon, C.E. y Weaver, W. 1949: The mathematical theory of communication. University of Illinois Press. Urbana Ill.

Silver, I.A. 1980: "La determinación de la edad en los animales domésticos". En D.R. Brothwell y E. Higgs (eds.): Ciencia en arqueología. Fondo de Cultura Económica. Madrid: 290-307.

Simpson, E.H. 1949: "Measurement of diversity". $\mathrm{Na}$ ture 163, 688.

Spearman, C.E. 1904: “'General intelligence' objectively determined and measured". American Journal of Psychology 15: 201-293.

Speth, J.D. y Clark, J. 2006: "Hunting and overhunting in the Levantine Late Middle Palaeolithic". Before Farming 3 (1): 1-42. 
Stiner, M.C. 1991: "Food procurement and transport by human and no-human predators". Journal of Archaeological Science 18: 455-482.

Stiner M.C., 1994: Honor among thieves: A zooarchaeological study of Neandertal ecology. Princenton University Press. Princenton.

Stiner, M.C. 2002: "On in situ attrition and vertebrate body part profiles". Journal of Archaeological Science 29: 979-991.

Straus, L.G. 1979: "Mesolithic adaptations along the northern coast of Spain". Quaternaria 21: 305-327.

Straus, L.G. 1992: Iberia before the Iberians. The Stone Age Prehistory of Cantabrian Spain. University of New Mexico Press. Alburquerque.

Straus, L.G. 1995: "Diversity in the face of adversity: Human adaptations to the environmental changes of the Pleistocene-Holocene transition in the Atlantic regions of Aquitaine, Vasco-Cantabria and Portugal'. En V. Villaverde (ed.): Los últimos cazadores. Transformaciones culturales y económicas durante el Tardiglaciar y el inicio del Holoceno en el ámbito mediterráneo. Instituto de Cultura Juan Gil-Albert y Diputación de Alicante. Alicante: 63-78.

Straus, L.G. 2004: "Transitions: Into and out of Mesolithic adaptations along the Atlantic Façade of Europe and beyond". En M.R. González Morales y G.A. Clark (eds.): The Mesolithic of the Atlantic Façade: Proceedings of the Santander Symposium. Anthropological Research Papers 55. Arizona: 249-260.

Straus, L.G. 2005: "The Upper Paleolithic of Cantabrian Spain". Evolutionary Anthropology 14: 145-158.

Straus, L.G. y González Morales, M.R. 2003: “The Mesolithic in the Cantabrian interior: fact or fiction?”. En L. Larsson, H. Kindgren, K. Knutsson,
D. Leoffler y A. Akerlund (eds.): Mesolithic on the Move. Oxbow Books. Oxford: 359-368.

Tomé, C. y Vigne, J.D. 2003: "Roe deer Capreolus capreolus age at death estimates: New methods and modern reference data for tooth eruption and wear, and for epiphyseal fusion". Archaeofauna 12: 157-173.

United States Department of Agriculture. Agricultural Research Service 2008: USDA National Nutrient database for Standard reference, Release 19, Nutrient Data Laboratory Home Page. http://www.nal.usda.gov/fnic/foodcomp/search.

Uzquiano, P. 1995: “'L'evolution de la vegetation a l'Holocene inicial dans le nord de l'Espagne a partir de l'étude anthracologique de trois sites archeologique". Quaternaire 6 (2): 77-83.

Vega del Sella, Conde de la 1916: Paleolítico de Cueto de la Mina (Asturias). Junta para la Ampliación de Estudios, Comisión de Investigaciones Paleontológicas y Prehistóricas, Memoria 13. Madrid.

Vega del Sella, Conde de la 1923: El Asturiense. Nueva industrial preneolítica. Junta para la Ampliación de Estudios, Comisión de Investigaciones Paleontológicas y Prehistóricas, Memoria 32. Madrid.

Von den Driesch, A. 1976: A Guide to the Measurement of Animal Bones from Archaeological Sites. Peabody Museum Bulletin 1. Peabody Museum. Harvard.

Zapata, L. 2000: La recolección de plantas silvestres en la subsistencia mesolítica y neolítica. Complutum 11: 157-169.

Zapata, L.; Ibáñez, J. y González Urquijo, J. 1997: “El yacimiento de la cueva de Kobaederra (Oma, Kortezubi, Bizkaia). Resultados preliminares de las campañas de excavación 1995-97'. Munibe 49: 51-63. 


\section{MEDIDAS OSTEOMÉTRICAS DE LA FAUNA DE LA CUEVA DE MAZACULOS II}

\section{Bos primigenius}

Nivel 3

Carpal I GB: 37,5

Carpal intermedio GB: 42,5

Carpal radial GB: 43,3

Carpal lunar GB: 47

Falange III DLS: 92,2 Ld: 75,2 MBS: 26,6 Ap: 32,5

Rótula GL: 74,5 GB: 62,5

GL: 73,5 GB: 60,3

Astrágalo Dm: 43,4 GLm: 72,1 Bd: 81,5 GLl: 52,2 Dl: 46 Dm: 43,8 GLm: 74,7 Bd: 52,4 GLl: 81,2 Dl: 46

Sesamoideo pequeño GB: $37,5 \mathrm{~W}: 15,9$

\section{Cervus elaphus}

Nivel 1.1

Carpal Intermedio GB: 31

Calcáneo GB: 29,6

Falange I Bp: 18,9 Bp: 18,6 SD: 13,7

Falange II Bp: 18,2 SD: 13,7

Nivel 3

Maxilar LP4 - M3: 71

$$
\text { L PD2 - PD4: } 48.8
$$

Metacarpo BFd: 37,8 Bd: 38,6

BFd: 36,2 Bd: 37,2

Carpal I GB: 23,5

Carpal intermedio GB: 36,5

Pelvis LA: 52 LAR: 47,9 SC: 31,6

Falange II GL 38,2 BP 19,6

BP 19,7

BP 22,5

Falange III DLS: 52,5 Ld: 49,8 MBS: 14,4 Ap: 17 DLS: 54,2 Ld: 51,1 MBS: 15,5 Ap: 17,6 DLS: 52,2

Astrágalo Dm: 27,8 Bd: 31,4

Centrotarsal GB: 47,9
Bos sp.

Nivel 3

Pd4 B: 13,7 L: 37,8 Sin desgaste

Metatarso Bp: 72 Bd: 73,1 SD: 40,6

Nivel A2

$\mathrm{M}_{3} \mathrm{~B}: 14,1 \mathrm{~L}: 32,6$

B: 12,2 L: 28,9

Escápula GLP: 40 LG: 52,1 BG: 34,6

Húmero BT: 53 SD: 27,4 Bd: 59

Radio Bd: 45,1

Bd: 43,5

Metacarpo Bp: 40,2

Carpal intermedio GB: 26,6

GB: 28,8

GB: 30

Carpal radial GB: 29

Carpal I GB: 28,1

Carpal accesorio GB: 20

Carpal ulnar GB: 20,4

GB: 20,6

Fémur DC: 35,5

Tibia Bd: 47,5

Maleolar GB: 25,5

Astrágalo Dm: 29,1 GLm: 49,3 Bd: $31 \mathrm{GL}: 153$ Dl: 27,7

Dm: 28,6 GLm: 47,2 Bd: 31,8 GLl: 50,6 Dl: 27,7

Dm: 28,7 GLm: 50,5 Bd: 31,7 GLl: 53,4 Dl: 28,3

GLl: 40,8 Dl: 26,1

Falange I Bp: 19,8

Bp: 17,3

Bp: 18,5

Falange II Bp: 17,8

Bp: 21

Bp: 19,3

Bp: 19,5

Bp: 19,7

Falange III DLS: 44,6 Ld: 41,9 MBS: 12,5 Ap: 15

DLS: $41,1 \quad$ MBS: 13,2 Ap: 16,5

Ap: 14,1

Centrotarsal GB: 40,5

Tarsal 2+3: 23,5

GB: 22,2

Sesamoideo GB: 13 L: 14, 8 W: 7,5 
Capreolus capreolus

Nivel 1

Carpal intermedio GB: 16,9

Nivel A2

$\mathrm{Pd}_{4}$ B: 6 L: 15,5 desgaste medio

Ulna LO: 38,3 DPA: 25 SDO: 21,8 BPC: 15,5

Falange II GL: 30,2 Bp: 12,5 SD: 9,7 Bd: 9,4

Fémur Atr: 22,5

Nivel 3

Escápula SLC: 21,4 GLP: 32,3 LG: 22,8 BG: 23

SLC: 19,4 GLP: 30,4 LG: 21,3 BG: 22,4

SLC: 19,4 GLP: 29,6 LG: 21,4 BG: 22

Radio Ap: 15,2

Bd: 26

Fémur: BP: 43,8 DC: 20,7

DC: 20

Bd: 41 Atr: 21,4

Pélvis: GL: 17 LA: 30 LAR: 26,2 SH: 19,8 SB: 8,1 Lfo:

26,2 SBI: 10,2

LA: 29,5 LAR: 25,1 SH: 20 SB: 9,7 Lfo

Astrágalo Dm: 18,4 GLm: 30,4 Bd: 20,2 GLl: 31,8 Dl: 18

Falange I Bp: 12,6

Falange II Bp: 11,2 SD: 9,9

Bp: 10,5

Sus scrofa/Sus sp.

Nível 1.1

Mandíbula LM: 220,5 BM: 216 LM: 340,2

Nivel 1.3

Falange II GL23,8 Bp17,6 SD14,2 Bd15,1

Nivel 3:

Húmero BT: 47,2

Rótula GL: 42,6 GB: 26,7

Astrágalo Dm: 26,4 GLm: 41,6 Bd: 25,4 GLl: 43,6 Dl: 23,8

Calcáneo GL: 85,2 GB: 22,6

Nivel A2:

Maxilar LP3-M1: 44

Escápula SLC: 29,1 GLP: 44,5 LG: 33,2 BG: 28,9 HS: 24

Húmero BT: 36,7

Centrotarsal GB: 29,1
Rupicapra rupicapra

Nivel A2

Radio BFp: 28,8 Bp: 31,4 Ap: 16,3

Ovicaprinos

$\underline{\text { Nivel A2 }}$

Pelvis LA (22)

Falange I GL: 34,7 Bd: 12,6
Vulpes vulpes

Nivel A2

Tibia GL: 123 Bp: 20 SD: 6,5 Bd: 12,5

Ursus arctos

Nivel A2

Falange II GL: 37 Bp: 19,8 SD: 14,2 Bd:14,2

Bp: 21

Meles meles

Nivel 3

Ulna LO: 12 DPA: 17 SDO: 14,5 BPC: 11,2

Erinaceus europaeus

Nivel 3

Húmero BT: 9,6 SD: 3.6 Bd: 13 